\title{
Dendritic Translocation Establishes the Winner in Cerebellar Climbing Fiber Synapse Elimination
}

\author{
Jennifer Carrillo, Naoko Nishiyama, and Hiroshi Nishiyama \\ Center for Learning and Memory, The University of Texas at Austin, Austin, Texas 78712
}

In many regions of the developing mammalian nervous system, functional synaptic circuitry is formed by competitive elimination of early formed redundant synapses. However, how winning synapses emerge through competition remains unclear in the brain largely because of the technical difficulty of directly observing this dynamic cellular process in vivo. Here, we developed a method of two-photon multicolor vital imaging to observe competitive elimination of supernumerary climbing fibers (CFs) in the cerebellum of live mouse pups. At birth, each Purkinje cell (PC) in the cerebellar cortex is innervated by multiple CFs; an activity-dependent regression of supernumerary CFs ultimately yields a single innervation for most PCs by postnatal day 21 . As supernumerary CFs are pruned, the terminal field of CFs translocates from the soma to the dendrites of PCs. In vivo time-lapse imaging of CF elimination revealed that (1) CF terminals were highly motile on the soma, but their motility was significantly reduced on dendrites; (2) only one CF could translocate to the dendrites whereas their competitors were restricted to perisomatic regions; and (3) the CF that began dendritic translocation became the winner. Moreover, selective photo-ablation of the winning CF (that undergoes dendritic translocation) reversed the fate of its losing competitor. These results indicate that dendritic translocation is a key cellular event that determines the winner during $\mathrm{CF}$ elimination. We propose that $\mathrm{CF}$ terminals are selectively stabilized on dendrites, providing irreversible competitive vigor to the first $\mathrm{CF}$ to form dendritic synapses.

\section{Introduction}

The developmental refinement of synaptic connections, through selective removal of early formed redundant axons, is crucial for generating functional synaptic circuitry in many regions of the nervous system (Purves and Lichtman, 1980; Katz and Shatz, 1996; Lichtman and Colman, 2000). At neuromuscular junctions (NMJs), elimination of redundant motor axons is triggered by asynchronous firing among competing inputs (Personius and Balice-Gordon, 2001; Personius et al., 2007), suggesting that timing of presynaptic and postsynaptic activity determines which inputs are retained (winner) versus eliminated (loser) through Hebbian-type mechanisms. Activity likely plays a role in winner/ loser determination across the nervous system (Katz and Shatz, 1996; Lichtman and Colman, 2000). However, it remains largely unclear how particular inputs impinging on the same postsynaptic neuron are selectively stabilized or eliminated at neuron-neuron synapses, especially in the CNS.

Unlike NMJs, synapses in the CNS form on the soma, dendrites, spines, and even occasionally on axons. Since each of these postsynaptic domains has distinct biochemical and electrical properties, axonal inputs might be regulated differently depend-

Received Sept. 24, 2012; revised March 16, 2013; accepted March 19, 2013.

Author contributions: H.N. designed research; J.C., N.N., and H.N. performed research; H.N. analyzed data; J.C. and H.N. wrote the paper.

This work was supported by University of Texas at Austin startup funds, Whitehall Foundation, and National Institutes of Health Grant NS073919 (H.N.). We thank Wesley Thompson, Jeremy Colonna, and Elise Shen for helpful comments on this manuscript.

The authors declare no competing financial interests.

Correspondence should be addressed to Hiroshi Nishiyama, Center for Learning and Memory, The University of Texas at Austin, 1 University Station Stop C7000, Austin, TX 7812. E-mail: hiroshi@utexas.edu.

DOI:10.1523/JNEUROSCI.4561-12.2013

Copyright $\odot 2013$ the authors $\quad 0270-6474 / 13 / 337641-13 \$ 15.00 / 0$ ing on their subcellular location on the postsynaptic surface. In fact, when supernumerary preganglionic inputs onto autonomic ganglion cells are pruned in the peripheral nervous system, somatic inputs are more likely eliminated than dendritic inputs. Moreover, the number of winners is related to dendritic complexity (Hume and Purves, 1981; Forehand, 1985). In the CNS, developmental pruning of axonal branches often occurs at the same time that synapses are changing their location from soma to dendrites or dendrites to spines (Palay and Chan-Palay, 1974; Crepel et al., 1976; Portera-Cailliau et al., 2005; Li et al., 2010). These observations raise the possibility that postsynaptic neurons use their distinct subcellular domains for selective maintenance/ removal of particular synapses during development.

To examine the role of neuronal subcellular domains in regulating synapse elimination, we used developmental regression of cerebellar climbing fibers (CFs) as a model. CFs, the terminal branches of axons originating in the inferior olive, provide excitatory glutamatergic drive to PCs in the cerebellar cortex. At birth, multiple CFs innervate the soma of each Purkinje cell (PC). The CF-PC synapses then undergo an activity-dependent refinement, such that by postnatal day 21 (P21), most PCs are innervated by a single CF on their dendrites (Crepel et al., 1976; Lohof et al., 1996; Kano and Hashimoto, 2009). Previous studies attempted to determine a role of this CF translocation from the soma to the dendrites in CF elimination (Sotelo, 1990; Scelfo et al., 2003; Hashimoto et al., 2009a). However, the results are controversial largely because the limitations of conventional experimental approaches: between-animal comparison of static images and physiological measurements without knowledge of the underlying synaptic structures. These approaches do not allow either conclusive identification of winning versus losing inputs or observa- 
tions of the sequential events of competition and elimination at the same synapses.

To overcome these limitations, we used in vivo microscopy for the first time to study developmental synapse competition in the CNS: multicolor time-lapse images of competing CFs were taken in live mice to examine the role of CF translocation in winner/ loser determination.

\section{Materials and Methods}

Tracer injection and cranial window preparation. All procedures were approved by the Institutional Animal Care and Use Committee of the University of Texas at Austin. Male and female C57BL/6 mice and transgenic (tg) mice that express enhanced green fluorescent protein (EGFP) under the control of neurofilament light chain (nefl) promoter (neflEGFP tg; Mutant Mouse Regional Resource Centers, 015882-UCD) were used for this study. In both mouse strains, CFs were labeled by olivary injection of a red fluorescent tracer, dextran-conjugated tetramethylrhodamine (TMR-dextran). The C57BL/6 mice were used for TMR singlecolor imaging (see Fig. $1 C$ ) and the nefl-EGFP tg mice were mostly used for EGFP/TMR dual-color imaging (see Figs. 3-6). The nefl-EGFP tg mice were also used for EGFP-single color imaging (see Figs. 1F, 2A), for which tracer injection (described below) was not performed.

Pups (P5-P7) of C57BL/6 mice or nefl-EGFP tg mice were anesthetized with an intraperitoneal injection of ketamine/xylazine $(40 / 4 \mathrm{mg} / \mathrm{kg})$ and placed in a stereotaxic device (Stoelting). The dorsal neck muscles were retracted to expose the dura over the foramen magnum and a small opening was then made in the dura to expose the brainstem. A glass pipette with a $10-20 \mu \mathrm{m}$ tip diameter was filled with mineral oil and inserted to a Nanoject II automated nanoliter injector (Drummond Scientific). The tip of the glass pipette was then front filled with $\sim 0.5 \mu \mathrm{l}$ of the TMR-dextran (Life Technologies) dissolved in water at a concentration of $0.8-1 \%$. Injections were made unilaterally at the midline, at the midpoint between the caudal edge of the cerebellar cortex and the $\mathrm{C} 1$ cervical vertebra, at a depth of $1.1 \mathrm{~mm}$. The pipettes were set at an angle of $60^{\circ}$ from vertical and $7^{\circ}$ from the midline toward the left inferior olive. A dye volume of $20-40 \mathrm{nl}$ was delivered over $5 \mathrm{~min}$. The pipettes were then left in place for $5 \mathrm{~min}$ before they were withdrawn.

Immediately after the tracer injection, the scalp overlaying skull and neck muscle were removed. Under a surgical microscope, the muscles and fascia overlaying the skull were removed and the skull surface was cleaned using a sterile cotton applicator. A small metal plate was then glued to the skull near lambda with surgical cyanoacrylate (Vetabond; $3 \mathrm{M}$ ) and dental cement (Fig. 1A). The mice were then transferred to a custom-made surgical stage. The animals' heads were securely held in place by clamping the metal plate on the skull with clamps attached to the stage. The occipital bone at this developmental stage is very thin and soft. Therefore, instead of a drill, a 26 gauge needle was used to etch a small rectangle $\left(\sim 2 \times 1.5 \mathrm{~mm}^{2}\right)$ on the skull over the part of the right cerebellar vermis and hemisphere. The bone inside of the rectangle was carefully lifted and removed by forceps to create a craniotomy. The degree of bleeding caused by cutting the bone varied from surgery to surgery. Although it mostly stopped naturally within $10-20 \mathrm{~min}$, a collagen sponge (Aviten Ultraform; Bard) was used to stop bleeding when necessary (Holtmaat et al., 2009). A coverslip was placed directly on top of the dura and the surgical cyanoacrylate was applied to the edge of the craniotomy to avoid bone regeneration. Dental cement was then applied around the coverslip and on the exposed skull surface to create a cranial window (Fig. $1 A, B)$. Isoflurane $(0.5-1.5 \%)$ was given if additional anesthesia was necessary. The animal was then allowed to recover from the anesthesia and returned to the home cage. Although $\sim 30-40 \%$ of mothers killed or neglected the pups after this surgery, pups gained weight and behaved indistinguishably from their unoperated littermates when they were accepted by mothers (their own or foster). Among the pups that survived, the chance to find at least one TMR-positive CF under the cranial window (i.e., successful tracer injection) was $\sim 80 \%$. Within the pups with successful tracer injection, the chance to find at least one PC that was innervated by both EGFP single-positive CF and EGFP/TMR double-positive CF was $\sim 60 \%$.
In vivo time-lapse two-photon microscopy. The animals were allowed at least $3 \mathrm{~d}$ of recovery after the surgery before they were lightly anesthetized with an intraperitoneal injection of ketamine/xylazine $(30 / 3 \mathrm{mg} / \mathrm{kg})$ and placed on a custom-made microscope stage. The head of the animal was securely held by clamping the metal plate on its head with a clamp attached to the stage. The stage was then fixed on an $x-y$ translator under a laser-scanning confocal microscope (FV1000MPE; Olympus) equipped with a $25 \times$ water-immersion objective lens (Olympus XLPlan N, 1.05 NA) and two external, nondescanned photomultiplier tubes (PMTs; R3896, Hamamatsu). For two-photon excitation of TMR (Fig. 1C) and simultaneous excitation of TMR/EGFP (Figs. 3-6), 870-890 nm of pulsed laser was provided by Mai Tai HP DeepSee mode-locked Ti:sapphire laser (Spectra-Physics). EGFP-only excitation (Figs. 1F, 2A) was performed at $920 \mathrm{~nm}$. The emitted green and red fluorescence was separated by a dichroic mirror (DM570) and then filtered by emission filters (bandpass filter 495-540 nm and 570-620 nm for green and red emissions, respectively) before they are detected simultaneously by the external PMTs. The $z$-stack images (spaced $1 \mu \mathrm{m}$ apart) of CFs in the cerebellar molecular layer were taken in vermal lobules 6-7. One to four different regions of interest (ROIs) were imaged per animal with high resolution (0.11-0.16 $\mu \mathrm{m} /$ pixel in most of the images).

In the developing cerebellar cortex, brain surface vasculature was not always a good landmark to locate the same ROIs for the time-lapse imaging, because surface vasculature was often too simple or the pattern changed as the cortex grew. Therefore, low-magnification images of TMR-containing CFs (CFs were sparse in the field of view) were taken with wide-filed fluorescence mode; the images were used to locate the same ROIs during the next imaging session. In case of EGFP single-color imaging, low-magnification two-photon images of CFs were taken (EGFP-positive CFs were abundant in the field of view) and the spatial pattern of the CFs was used to locate the same ROIs (Fig. $1 F$ ). After image acquisition, the animal was allowed to recover from the anesthesia and returned to the home cage.

Selective photo-ablation. It has been shown that all or part of a single neuron can be selectively killed by filling the neuron with fluorescent dye and strongly exciting the dye (Miller and Selverston, 1979; Jacobs and Miller, 1985; Camhi and Macagno, 1991; Warzecha et al., 1992). We therefore reasoned that if TMR or other dextran-conjugated fluorescent dye was selectively overexcited without exciting EGFP, the CFs containing the dye could be selectively ablated without affecting nearby EGFP single-positive CFs. Tracer injection and cranial window preparation was performed on nefl-EGFP tg mice as described in the previous section, except that a mixture of TMR-dextran $(0.8-1 \%)$ and Alexa Fluor 594 (A594)-dextran (0.8-1\%; Life Technologies) was used instead of TMRdextran alone. The CFs labeled by this mixture became A594/EGFP/TMR triple-positive. However, A594-dextran alone was injected in the experiment shown in Figure 6A. Therefore, the red CF in Figure 6A was A594/ EGFP double-positive.

The A594, like TMR, emits orange-red fluorescence and we used it because A594 and EGFP have quite different two-photon excitation properties. Two-photon action cross section (the product of the twophoton absorption cross section and the fluorescent quantum yield) of EGFP is $\sim 80$ and $40 \mathrm{GM}$ at 890 and $830 \mathrm{~nm}$, respectively (Zipfel et al., 2003). On the other hand, it is $\sim 2$ and 65 GM for A594 at 890 and 830 $\mathrm{nm}$, respectively (manufacturer's Web site). Therefore, when excited at $890 \mathrm{~nm}$, EGFP is estimated to have $\sim 40$ times more fluorescence than A594, if the same amounts of EGFP and A594 molecules are present. However, as shown in Figure 6 A, right, EGFP was just slightly $(\sim 2 \times)$ brighter than A594 at $890 \mathrm{~nm}$, indicating that the number of A594 molecules in the CFs was significantly more $(\sim 10-20$ times $)$ than that of EGFP molecules. Thus, when excited at $830 \mathrm{~nm}$ (where A594 has a higher two-photon action cross-section than EGFP), A594 became overwhelmingly brighter than EGFP (Fig. $6 \mathrm{~A}$, left). It should be noted that much less laser power was required at $830 \mathrm{~nm}$ to excite A594, because the twophoton action cross section of A594 at $830 \mathrm{~nm}$ is $~ 30$ times larger than that at $890 \mathrm{~nm}$ (Fig. 6A).

As we expected, this selective overexcitation of A594 at $830 \mathrm{~nm}$ enabled selective photo-ablation of A594-containing CFs (Fig. 6B-D). It was not a result of nonselective damage caused by high-power laser because (1) 
less laser power was used for the overexcitation of A594 than that used for multicolor time-lapse imaging and (2) nearby EGFP single-positive CFs (i.e., those not containing A594) were not damaged at all (Fig. 6B-D).

In vivo time-lapse imaging with photo-ablation was performed as follows. (1) Simultaneous multicolor imaging was performed at 870-890 $\mathrm{nm}$, as described in the previous section. (2) If an A594/EGFP/TMR triple-positive CF (yellow or orange) and an EGFP single-positive CF (green) competed for the same PC and the yellow/orange CF was dominant, the wavelength was switched to $830 \mathrm{~nm}$ (average power, $\sim 30 \mathrm{~mW}$ ). Then, $z$-stack images of the CFs were taken with the exact same ROI $\left(63 \times 63-84 \times 84 \mu \mathrm{m}^{2}\right), z$-spacing $(1 \mu \mathrm{m})$, and stack thickness $(30-50$ $\mu \mathrm{m})$ used to take the $z$-stack images of the same CFs at $870-890 \mathrm{~nm}$ in the previous step. The entire duration of A594 overexcitation by this procedure was $\sim 50-80 \mathrm{~s}$ (depending on the thickness of the $z$-stack). After the overexcitation, the animal was allowed to recover from the anesthesia and returned to the home cage. (3) Multicolor time-lapse imaging at $870-890 \mathrm{~nm}$ was then resumed from the next imaging session.

Electrophysiology. Under deep anesthesia with 5\% isoflurane, neflEGFP tg mice (P11-P12) were decapitated and the cerebellum was rapidly dissected into ice-cold low-sodium saline containing the following (in mM): 205 sucrose, $2.5 \mathrm{KCl}, 0.5 \mathrm{CaCl}_{2}, 7 \mathrm{MgCl}_{2}, 25 \mathrm{NaHCO}_{3}, 1.25$ $\mathrm{NaH}_{2} \mathrm{PO}_{4}$, and 20 dextrose, equilibrated with $95 \% \mathrm{O}_{2}$ and $5 \% \mathrm{CO}_{2}$. Parasagittal slices of cerebellar vermis $(250 \mu \mathrm{m}$ thick $)$ were prepared in the same solution using a Microm HM650V (Thermo Scientific) vibrating tissue slicer. Slices were recovered in artificial CSF (ACSF) containing the following (in mM) $125 \mathrm{NaCl}, 2.5 \mathrm{KCl}, 2 \mathrm{CaCl}_{2}, 1 \mathrm{MgCl}_{2}, 25 \mathrm{NaHCO}_{3}$, $1.25 \mathrm{NaH}_{2} \mathrm{PO}_{4}$, and 20 dextrose, equilibrated with $95 \% \mathrm{O}_{2}$ and $5 \% \mathrm{CO}_{2}$. After a recovery period of at least $1 \mathrm{~h}\left(\right.$ at $32^{\circ} \mathrm{C}$ during the initial $30 \mathrm{~min}$ and then at room temperature thereafter), slices were placed in a submerged recording chamber that was perfused with ACSF supplemented with $5 \mu \mathrm{M}$ gabazine to block $\mathrm{GABA}_{\mathrm{A}}$ receptors. The patch electrodes (with resistance of $\sim 3 \mathrm{M} \Omega$ ) were filled with a solution containing the following (in mM): $35 \mathrm{CsF}, 100 \mathrm{CsCl}, 10 \mathrm{HEPES}$, and $10 \mathrm{EGTA}, \mathrm{pH} 7.3$ was adjusted with $\mathrm{CsOH}$. At room temperature, whole-cell voltageclamp recordings of CF-evoked EPSCs (CF-EPSCs) were made from visually identified PCs located at the top of folia (gyrus) in lobule 6-7 (a brain region under our cranial window). Ionic currents were recorded with an Axon Multiclamp 700B patch-clamp amplifier (Molecular Devices). CFs were stimulated by applying paired pulses (duration $200 \mu \mathrm{s}$, interval $100 \mathrm{~ms}$ ) through a glass pipette (2-3 $\mu \mathrm{m}$ tip diameter) filled with ACSF. CF-EPSCs were identified by their all-or-none response and paired-pulse depression. To search for multiple CF innervations, the stimulation pipettes were systematically moved in the granule cell layer and the stimulus amplitude was gradually increased over the range of $0-90 \mathrm{~V}$ at each stimulation site. These data were acquired (filtered at 2 $\mathrm{kHz}$ and digitized at $20 \mathrm{kHz}$ ) and analyzed using AxoGraph X (AxoGraph) software.

Calcium imaging. A mixture of TMR-dextran (0.8-1\%) and Oregon Green BAPTA-1 (OGB1; $\mathrm{Ca}^{2+}$ indicator)-dextran (5\%) was injected into the inferior olive of nefl-EGFP tg mice as described in the previous section. The CFs labeled by this mixture became TMR/OGB1/EGFP triple-positive. After a survival period of at least $2 \mathrm{~d}$, parasagittal slices of cerebellar vermis were prepared at P9-P10 and CF-EPSCs recordings were performed as described in the previous section. Before performing whole-cell recordings, simultaneous multicolor imaging was performed at $870-890 \mathrm{~nm}$ to identify PCs that were co-innervated by TMR/OGB1/ EGFP triple-positive CF and EGFP single-positive CF. Whole-cell recordings were made from such PCs and stimulation pipettes were placed on the TMR/OGB1/EGFP triple-positive CFs (yellow or orange) in the granule cell layer. CF-EPSC recordings were synchronized with $\mathrm{Ca}^{2+}$ imaging. Fluorescent changes of OGB1 upon CF stimulation were imaged at a $10 \mathrm{~Hz}$ acquisition rate with single-photon excitation by the 473 $\mathrm{nm}$ line of a diode laser coupled to the FV1000MPE laser-scanning confocal microscope. Since OGB1-containing CFs also contain EGFP, green fluorescence of OGB1 was detected together with that of EGFP. Although it significantly reduced relative changes of green fluorescence $\left(\Delta F / F_{0}\right)$ upon $\mathrm{Ca}^{2+}$ increase, $\mathrm{CF}$-evoked $\mathrm{Ca}^{2+}$ signals were clearly detected when the signals were averaged $2-3$ times.
Image analysis. The images were transferred to National Institutes of Health ImageJ (http://rsb.info.nih.gov/ij/) and processed with a median filter ( 2 pixel radius) to reduce shot noise in each optical plane. For comparing structural stability of somatic and dendritic terminals, CF varicosities were identified in raw $z$-stacks and numbers of stable, new (i.e., seen for the first time), and disappeared varicosities over $1 \mathrm{~d}$ intervals were counted. To facilitate comparison between two time points, a small number of images, typically consisting of $<5$ optical planes, were extracted from the $z$-stacks and maximally projected. At each time point, 3-5 such maximum projections were created to cover the soma and proximal portion of primary dendrites and they were used as an initial comparison. However, the raw $z$-stacks were always examined for confirmation. To distinguish soma and dendrites, $3 \mathrm{D}$ projections of the same CFs were reconstructed from the $z$-stacks to yield sagittal views. All somatic varicosities and dendritic varicosities located $\sim 5-15 \mu \mathrm{m}$ above the soma were quantified.

For time-lapse imaging of CF competition and dendritic translocation, PCs innervated by two CFs were identified in raw $z$-stacks. Sagittal views of the same CFs were reconstructed by $3 \mathrm{D}$ projections to identify dendritic translocation. Fluorescent signals from neighboring CFs often prevented reconstructing clear 3D projections of CFs of interest. In such cases, the neighboring CFs were removed from the original $z$-stacks before creating $3 \mathrm{D}$ projections.

For quantifying relative innervation areas of two competing CFs at the PC soma, multicolor images obtained in the calcium imaging experiment and multicolor in vivo time-lapse images taken before dendritic translocation proceeded were used. Approximately 10 optical planes covering the soma were extracted from the $z$-stacks and maximally projected. Since a morphologically dominant CF in the raw $z$-stacks was always dominant in the maximum projection as well, the projected images were used for the quantification. In our multicolor images, fluorescent signals in a red detection channel solely consist of yellow CF (i.e., TMRcontaining $\mathrm{CF}$ ), whereas signals in a green detection channel consist of a mixture of yellow and green CFs (because TMR-containing CF also contains EGFP, thereby detected by a green detection channel as well). Therefore, to separate the green CF from the yellow CF, red fluorescence was subtracted from green fluorescence by using the Image Calculator function of ImageJ. Next, by using the Local Threshold plug-in of ImageJ, fluorescent signals that represent each color of CF were separated from background signals, and the number of pixels $(\mathrm{N})$ contained by each $\mathrm{CF}$ on the soma were counted. Relative innervation area of each CF (green or yellow) was calculated as $\mathrm{N}$ (each)/ $\mathrm{N}$ (total). Here, $\mathrm{N}$ (each) is $\mathrm{N}$ (green $\mathrm{CF}$ ) or $\mathrm{N}$ (yellow $\mathrm{CF}$ ), and $\mathrm{N}$ (total) is $\mathrm{N}$ (green $\mathrm{CF}$ ) $+\mathrm{N}$ (yellow $\mathrm{CF}$ ). As shown in Figure 5, relative innervation area calculated by this method correlated well with their relative synaptic strength.

Immunohistochemistry. Pups (P9-P13, and P19) of nefl-EGFP tg mice were anesthetized with an intraperitoneal injection of ketamine/xylazine $(60 / 6 \mathrm{mg} / \mathrm{kg}$ for P9-P13, 100/10 mg/kg for P19) and perfused intracardially with $4 \%$ paraformaldehyde in PBS. Sagittal slices (50 $\mu \mathrm{m}$ thick) of the cerebellum were stained with rabbit polyclonal antibody against GFP (1:1000; Life Technologies) and guinea pig polyclonal antibody against vesicular glutamate transporter 2 (VGluT2; 1:200; Millipore; Fig. 2C), or mouse monoclonal antibody against calbindin-D-28K (1:2000; Sigma-Aldrich; Fig. 1D). For quantifying GFP-positive neurons in the inferior olive, coronal slices ( $30 \mu \mathrm{m}$ thick) of the medulla were stained with rabbit polyclonal antibody against GFP (described above) and mouse monoclonal antibody against NeuN (1:250; Millipore, Fig. 1E). The primary antibodies were detected by Alexa Fluor 488-labeled goat anti-rabbit antibody, Alexa Fluor 594-labeled goat anti-guinea pig antibody, and Alexa Fluor 546labeled goat anti-mouse antibody (all 1:1000; Life Technologies). The confocal image stacks (spaced $1.5 \mu \mathrm{m}$ apart) were acquired using a laserscanning confocal microscope with single-photon excitation.

\section{Results}

\section{Imaging $\mathrm{CF}$ development in vivo}

CF elimination is divided into two mechanistically distinct phases (Kano and Hashimoto, 2009). Several redundant CFs are eliminated in the initial phase before dendritic translocation, and 

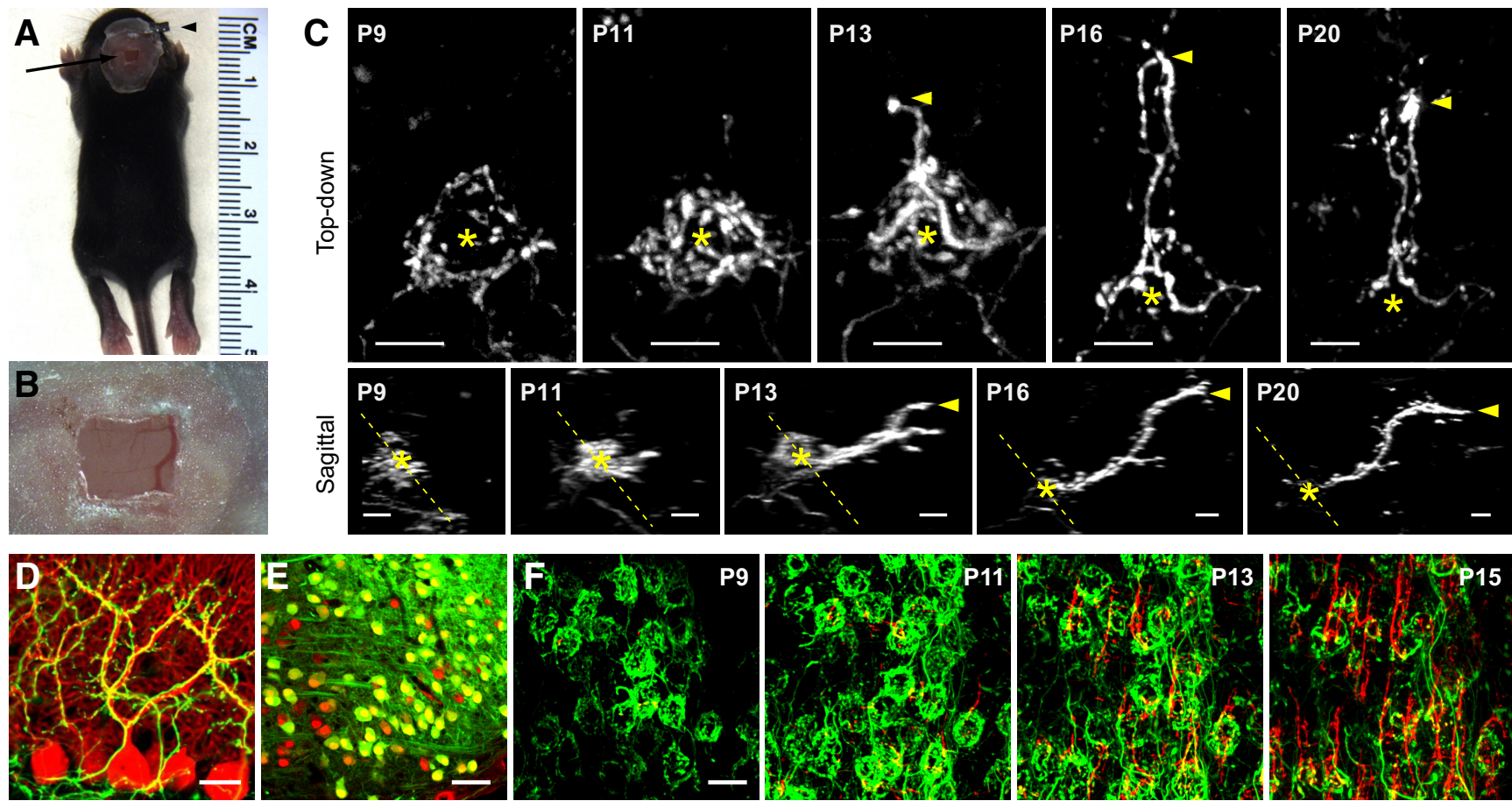

Figure 1. Time-lapse in vivo imaging of $C F$ dendritic translocation. $A, A$ mouse pup (P10) $3 \mathrm{~d}$ after the surgery. An arrow indicates the small rectangular shaped window. An arrowhead indicates the metal plate used to hold the head still in the anesthetized animals. B, A higher magnification view of the cranial window shown in $\boldsymbol{A}$. C, The same CF innervating a single $\mathrm{PC}$ repeatedly imaged in vivo by two-photon microscopy during the period of late phase CF elimination. CF was labeled by TMR-dextran. Top, Maximum projections showing top-down views of the CF. Bottom: Sagittal views of the same CF reconstructed from the same image stacks collected for the top panels. A whole CF tree (i.e., a terminal portion of olivocerebellar axon between the $\mathrm{PC}$ layer and pial surface) is shown in both the top-down and sagittal views. Asterisks indicate approximate location of the P( soma innervated by the CF. Arrowheads indicate corresponding points on the dendrites in the top and the bottom panels. In the bottom panels, dashed lines indicate approximate location of the PC layer and pial surface is located at the right side of the images (hence, the CF extends toward right of the images as the dendritic translocation proceeds). Scale bars: $10 \mu \mathrm{m}$. Note that (1) the scale differs between the top and the bottom panels and (2) the primary dendrites of the PC innervated by the CF were not entirely parallel to the optical axis. Therefore, in the top panels, the CF appears to extend away from the soma (toward top of the images) as the dendritic translocation proceeds. The (F began dendritic translocation at P11 (i.e., extended its arbor toward right in the bottom panels). At P13, the (F innervated both the soma and dendrites of the PC. The somatic innervation became less prominent afterward (see the image at P16) and mostly disappeared by P20, whereas the dendritic innervation persisted. D, Fixed cerebellar slices of nefl-EGFP tg mice (P19) were immunostained with anti-GFP (green) and anti-calbindinD-28K antibody (a marker for PC; red). Note: (Fs are the only GFP-positive structures in the cerebellar molecular layer. Scale bar, $10 \mu \mathrm{m}$. $\boldsymbol{E}$, Fixed slices of inferior olive derived from nefl-EGFP tg mice (P12) were immunostained with anti-GFP (green) and anti-NeuN antibody (a neuronal marker; red). Note: Most olivary neurons are GFP positive. Scale bar, $20 \mu \mathrm{m} . \boldsymbol{F}$, Time-lapse in vivo imaging performed with an nefl-EGFP tg mouse (without tracer injection). Maximum projections showing top-down views of the CFs are shown. The same imaging field was approximately located by approximate location in the cranial window and then confirmed by low-magnification two-photon images. As shown in this example, the spatial pattern of EGFP-positive CFs is a reliable landmark to locate the same set of CFs for time-lapse imaging. These projections are color coded such that optical planes above PC soma are red and others (planes below and at $\mathrm{PC}$ soma) are green. Therefore, red color indicates the (F portions translocating to the dendrites. Scale bar, $20 \mu \mathrm{m}$.

the remaining one or two redundant CFs are subsequently eliminated in the late phase while dendritic translocation proceeds. In this study, we focused on the late phase elimination to examine the role of synaptic location (soma vs dendrites) in determining winner vs loser.

Late phase elimination starts approximately mid second postnatal week (Kano and Hashimoto, 2009). To label nascent CFs, we first used TMR-dextran, a red color anterograde fluorescent tracer. We injected the TMR-dextran into the inferior olivary nucleus (origin of CFs) of mouse pups (P5-P7), and immediately after the injection, prepared a chronic cranial window over the cerebellar vermal lobules 6-7 (Fig. 1A,B) because this region is accessible with a conventional objective lens. At least $3 \mathrm{~d}$ after the surgery, CFs labeled by TMR were imaged in the cerebellar molecular layer of anesthetized mice (the pups were between P8 and P11 at the first imaging session). CFs were first identified under the wide-field fluorescence observation and then two-photon microscopy was used to take high-resolution images. The same CFs were repeatedly imaged thereafter every 1-3 d (mostly $2 \mathrm{~d}$; Fig. 1C). Although chemical (nonprotein) fluorescent tracers have rarely been used for chronic in vivo imaging, we found that the TMR signals were usually stable for $>10 \mathrm{~d}$, and thus sufficient to visualize the entire period of late phase CF elimination.

At P8-P9, the terminal arbors of labeled CFs formed an aggregate of varicosities around the basal portion of PC soma (Fig. $1 C$ ), consistent with the previously characterized morphology of developing CFs called the "pericellular nest" (Ramon y Cajal, 1911). By P10-P11, most CF terminals had moved to the apical portion of PC soma forming a "capuchon" type plexus (Ramon y Cajal, 1911), and dendritic translocation usually began at this stage (Fig. $1 C$, bottom). This onset of dendritic translocation was slightly (1-2 d) slower than a previous study using mice (Hashimoto et al., 2009a). To investigate the possibility that a potential damage to the olivary neurons by tracer injection altered the development of CFs, we used nefl-EGFP tg mice in which most CFs were labeled by EGFP. Although EGFP expression was widely seen in the CNS of the tg mice, it was confined to CFs in the cerebellar molecular layer (Fig. 1D). Approximately $75 \%$ (286 of 383 neurons) of olivary neurons express EGFP in the caudal part of the medial accessory olive [a subnucleus of the inferior olive that is projecting CFs to the cerebellar vermis (Sugihara et al., 2001; Figure 1E)]. Therefore, we estimated that a 
similar fraction (70-80\%) of CFs were EGFP positive under our cranial window. Time-lapse in vivo images of the EGFP-positive CFs showed that the overall dynamics of dendritic translocation were similar to those observed in the tracer-injected mice (Fig. $1 F)$. Moreover, dendritic translocation was rarely observed in lobules 6-7 at P9 in a perfusion-fixed brain without any prior surgery (data not shown). These results indicate that neither tracer injection nor chronic cranial window affects onset of CF dendritic translocation. Since CF development in lobules 6-7 is slower than in other vermal lobules (Sugihara, 2005), the slight difference in the timing of dendritic translocation between this study and the previous study (Hashimoto et al., 2009a) was likely due to the difference in cerebellar regions used. In lobules 6-7, dendritic translocation began after P9, proceeded rapidly after P11, and completed mostly by P20 (Fig. 1C,F).

\section{Somatic and dendritic CF terminals have different structural stability}

All somatic CF terminals are transient. They move from basal to apical portions of the soma and eventually disappear as dendritic translocation proceeds (Ramon y Cajal, 1911). However, stability of nascent dendritic CF terminals remains unknown. To determine whether dendritic translocation affects structural stability of CF terminals, we used the nefl-EGFP tg mice and the same CFs were repeatedly imaged at P11 and 12 or at P12 and 13. We then quantified the fraction of CF varicosities that appeared or disappeared over $1 \mathrm{~d}$ on both the soma and dendrites of the same PCs. Consistent with the fact that somatic CF terminals are transient, $58 \%$ of somatic CF varicosities appeared or disappeared over the course of $1 \mathrm{~d}$ (Fig. $2 A, B$ ). On the other hand, varicosities on the proximal portion of dendrites, located only 5-15 $\mu \mathrm{m}$ above the soma, were largely stable: only $20 \%$ of them appeared or disappeared (Fig. $2 A, B)$. This difference was statistically significant $(p<0.01$, $\chi^{2}$ test). Since more than half of PCs in lobules $6-7$ are already innervated by single CFs at this developmental stage (Fig. 3D) and the difference between somatic and dendritic CF varicosities was observed in all eight PCs analyzed (Fig. 2B), these data strongly suggest that the structural stability of CF varicosities primarily depends on their subcellular location on PCs, rather than their presynaptic cell of origin.

To confirm that EGFP-positive varicosities were functional CF terminals, we performed GFP and VGluT2 double immunostaining (Fig. 2C). VGluT2 is used as a marker for functional CF terminals in both immature and mature animals (Fremeau et al., 2001; Ichikawa et al., 2002; Hashimoto et al., 2009a). Moreover, a previous study using immunoelectron microscopy showed that VGluT2-positive CF varicosities were located at synapses on both the soma and dendrites during this developmental stage (Hashimoto et al., 2009a). Since VGluT2 is not expressed in the axonal backbone, we focused on varicosities (that have at least two times more diameter than adjacent processes) and quantified the fraction of EGFP-positive varicosities that also express VGluT2. Approximately $87 \%$ of EGFP-positive varicosities were VGluT2 positive on both soma (145 of 167 varicosities) and dendrites (68 of 78 varicosities), indicating that EGFP-positive varicosities were mostly functional CF terminals regardless of their subcellular location. These results collectively indicate that translocating CF terminals are stabilized on dendrites.

\section{Multicolor labeling of competing CFs}

Single color imaging, either with TMR (red) or EGFP (green), does not reveal whether the observed CFs are single CFs or mul-
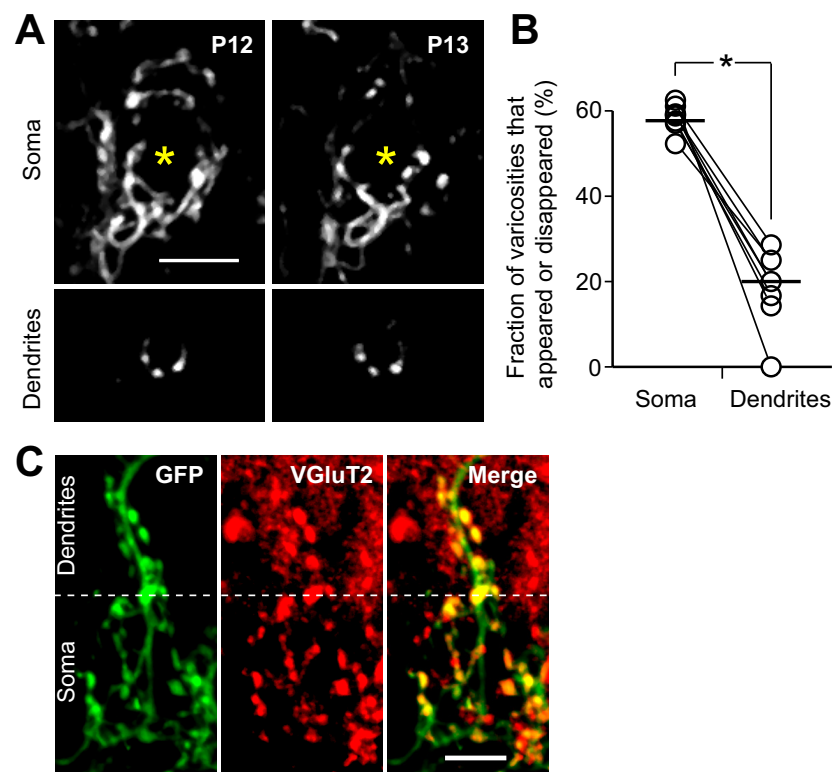

Figure 2. Dendritic CF terminals are more stable than somatic terminals. $\boldsymbol{A}$, Time-lapse images of CFs labeled by EGFP in a nefl-EGFP tg mouse. CF varicosities located on the soma and primary dendrite of the same $P C$ are compared. Maximum projections containing most of the somatic varicosities (top two images) and the $3 \mu \mathrm{m}$ segment of the proximal primary dendrite (bottom two images) $\sim 8-10 \mu \mathrm{m}$ above the soma are shown. Scale bar, $10 \mu \mathrm{m}$. $\boldsymbol{B}$, The fraction of varicosities that appeared or disappeared over $1 \mathrm{~d}$ was compared between soma and dendrites in each PC. A pair of open circles connected by a line represents the data from a single PC. Horizontal bars represent the average of eight analyzed PCs. Asterisk denotes $p<0.01$ ( $\chi^{2}$ test). Note that each PC is innervated by a single $(F$ in $>50 \%$ of the cases. Hence, these data indicate that even the varicosities of single (Fs are more stable on the dendrites than the soma. C, Fixed cerebellar slices of nefl-EGFP tg mice (P13) were immunostained with anti-GFP and anti-VGluT2 antibody. White dashed lines indicate the approximate location of the border between the soma and proximal dendrites. Most EGFP-positive CF varicosities were also VGluT2 positive on both the soma and dendrites, indicating that they are functional terminals. Scale bar, $10 \mu \mathrm{m}$.

tiple CFs intermingling with each other. To image how CF competition and elimination dynamically proceeds, multiple CFs competing for the same PCs must be labeled with different colored fluorophores as has been done at the NMJ (Walsh and Lichtman, 2003). To achieve this, we injected a small amount of TMR-dextran into the inferior olive of the nefl-EGFP tg mice. The underlying idea was that the majority of CFs would be EGFP single positive and that a minority of CFs would be EGFP/TMR double positive and could be distinguished from each other when the green and red fluorescence was simultaneously excited and detected. A similar strategy was successfully used to distinguish two competing CFs in fixed cerebellar slices by staining VGluT2 single-positive CFs (majority) and VGluT2/tracer double-positive CFs (minority) after an olivary injection of a small amount of dextran-conjugated tracer (Hashimoto et al., 2009a).

By using two-photon excitation at $870-890 \mathrm{~nm}$, we imaged EGFP and TMR signals simultaneously in vivo (Fig. $3 A-C$ ). Most CFs were EGFP single positive (Fig. $3 A$ ), but a small fraction of them were colabeled by TMR (Fig. $3 B$ ). The EGFP/TMR doublepositive CFs became yellow (or orange, depending on the amount of TMR present) when the green and red channels were merged. In some cases, green and yellow CFs innervated the same PC showing that our dual-color labeling can identify two competing CFs in live mouse pups (Fig. 3 C). To examine whether two colors are sufficient to distinguish all individual CFs competing for the same PCs during late phase elimination, we quantified the number of CF innervations per PC. Whole-cell voltage-clamp record- 

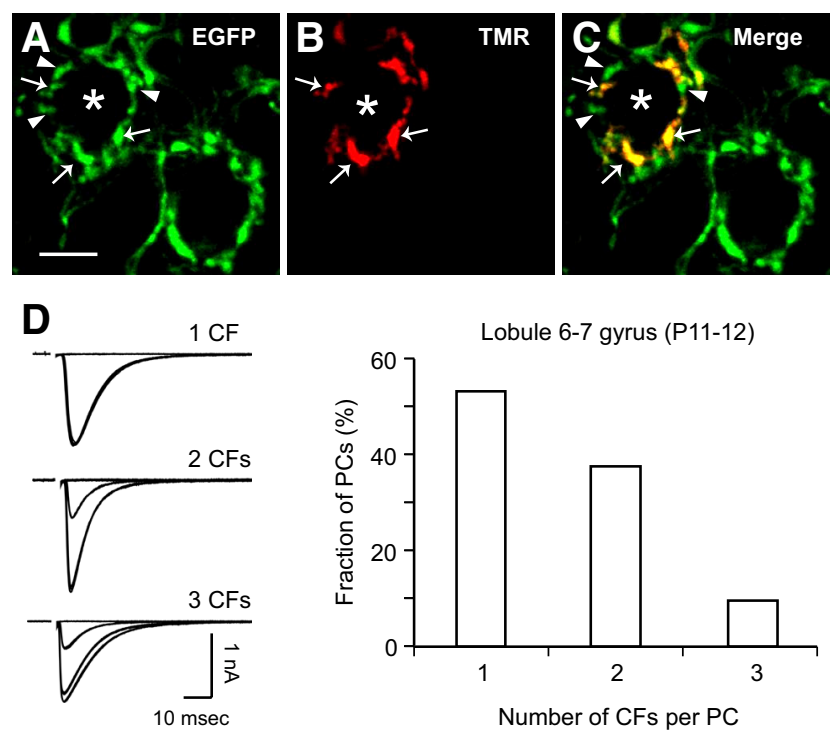

Figure 3. Multicolor in vivo imaging of two (Fs competing for a same $P C$. $A-C$, The left $P C$ (asterisks) is innervated by both a EGFP single-positive (F (arrowheads in $\boldsymbol{A}$ and $\boldsymbol{C}$ ) and a EGFP/ TMR double-positive (F (arrows in $\boldsymbol{A}-\boldsymbol{C}$ ) at $\mathrm{P}$. The middle and right $\mathrm{PC}$ are innervated by only EGFP single-positive CFs. Single Z-sections (not maximum projections) are shown here for validating the multicolor labeling. Scale bar, $10 \mu \mathrm{m}$. D, Two colors are mostly sufficient to distinguish all individual CFs competing for the same PC during late phase CF elimination. Left, Representative traces of (F-EPSCs innervated by single (top), double (middle), and triple (bottom) CFs. CF-EPSCs, evoked by gradually increased stimulus intensity, were overlaid. Holding potential was $-20 \mathrm{mV}$. Right, A histogram showing fractions of PCs innervated by single, double, and tripe CFs. Approximately $90 \%$ of PCs were innervated by single or double CFs at P11-P12.

ings were made from the somata of PCs in parasagittal slices derived from juvenile (P11-P12) mice. CF-EPSCs were recorded from PCs located at the top of folia (gyrus) in lobule 6-7. CFEPSC is elicited in an all-or-none manner. Thus, the number of discrete EPSC steps as the stimulus intensity is gradually increased indicates the number of CF innervations per PC recorded (Kano et al., 1995). Of 32 PCs recorded, 29 PCs (91\%) were innervated by no more than two CFs (Fig. 3D). Therefore, if a PC is innervated by green and yellow CFs after P11-P12, each color of CF represents a single CF in $90 \%$ of the cases.

\section{Multicolor in vivo time-lapse imaging of CF competition and elimination}

Multicolor vital imaging began at P8-P11 (most animals were P9-P10). Time-lapse images were taken only when green and yellow CFs innervated the same PCs (i.e., two competing CFs were visually distinguishable) at the first time point (such as Fig. $3 A-C$ ), and the same competing CFs were repeatedly imaged thereafter every 2-3 d until CF single innervation (determined morphologically) was completely or mostly established (Fig. 4). We obtained time-lapse images from 45 PCs (from 22 mice) but analyzed them only when two CFs, labeled by different colors, still innervated the same PCs at P11 or P12. As explained above, application of this criterion provided some assurance that each color represented a single CF. Thirty-five PCs (from 17 mice) satisfied the criterion and CF single innervation was established by P15 in most of the cases (24 PCs, 69\%). Four PCs (11\%) remained innervated by two CFs even after P19. These results are largely consistent with previous electrophysiological studies that characterized time course of late phase CF elimination in mice (Kano et al., 1995, 1997; Offermanns et al., 1997). Thus, our multicolor labeling and imaging tech- niques are suitable to analyze the dynamic progression of late phase CF elimination in vivo.

With this in vivo time-lapse approach, we first sought to determine conclusively whether one or multiple CFs translocate to dendrites of the same PCs during late phase elimination. Previous studies using in vitro electrophysiology and functional imaging yielded conflicting results regarding this question. Scelfo et al. (2003) performed simultaneous CF-EPSCs recording and $\mathrm{Na}^{+}$ imaging from the PCs innervated by multiple CFs and found that activation of each of the competing CFs evoked a distinct and localized $\mathrm{Na}^{+}$signal on the dendrites. Since the $\mathrm{Na}^{+}$signal most likely represents a CF innervation field under their experimental conditions, their results indicate that multiple competing CFs translocate to the dendrites (Scelfo et al., 2003). On the other hand, Hashimoto et al. (2009a) analyzed the kinetics of CFEPSCs and quantal CF-EPSCs (qEPSCs; arising from single synaptic vesicle in CF terminals) and found that stronger (presumably winning) CFs and weaker (presumably losing) CFs have slower and faster kinetics, respectively. Since the kinetics of CF-EPSCs and qEPSCs correlated with the distance between CF innervation field and recording electrode placed on the soma (slower the kinetics, more distant the CF from the soma), their results indicate that only a strong (winning) CF undergoes dendritic translocation (Hashimoto et al., 2009a). The reason underlying this clear discrepancy between those two studies remains unclear, but $\mathrm{Na}^{+}$signals might diffuse away from the synaptic sites and slower versus faster kinetics of CF-EPSCs and qEPSCs might not always represent somatic versus dendritic innervations. Although the claim of Hashimoto et al. (2009a) is supported by electron microscopy, only a few PCs were reconstructed at each time point (P9, 12, and 15).

Direct visualization of the competing CFs and observation of their dendritic translocation in vivo is arguably the best experimental approach to address this controversy. Our time-lapse images revealed that only one CF could translocate to the dendrites whereas its competitor was restricted to perisomatic regions in 34 of the 35 PCs (97\%) that satisfied the criterion described above (Fig. 4). Only in one PC, two CFs translocated to the dendrites and the PC remained innervated by the two CFs even at P21, suggesting that dual-CF dendritic translocation is a rare event that results in incompletion of synapse elimination (Fig. 4E). In the other 34 PCs, the CF that translocated to the dendrites always became the winner. One remarkable example can be seen in Figure $4 A$. Although one CF (yellow) appeared to dominate its competitor (green) on the soma, the green CF was the one that began dendritic translocation. While the green CF kept growing along the dendritic tree, the yellow CF was confined to somatic surface until P16 and never translocated to dendrites. The yellow CF had mostly disappeared by P19, leaving just a few varicosities on the soma. Except for the one PC shown in Figure $4 E$, a $C F$ that underwent dendritic translocation was never replaced by its competitor. To quantify this, we measured the height of CFs in the molecular layer (i.e., the distance along the optical axis from the most superficial point of the $\mathrm{CF}$ to the top of the soma) and compared it between the winner and loser in all 35 PCs analyzed. Although CF single innervation was not established by the last time point in four PCs, one CF was clearly dominant (i.e., innervated most of the dendritic surfaces) in all of these cases, thus it was considered the winner. As shown in Figure $4 F$, the difference between winner and loser was already significant $(p<0.001$, Mann-Whitney $U$ test) at early time points (P10-P12), indicating that a CF's fate is determined by whether or not it begins dendritic transloca- 

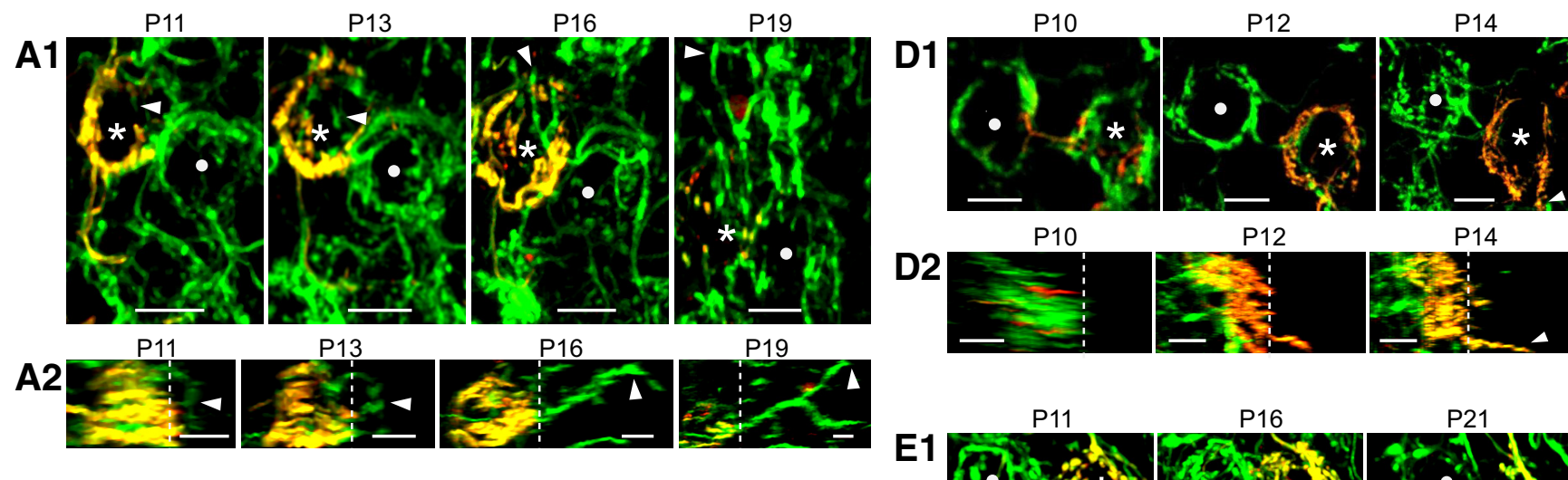

P12

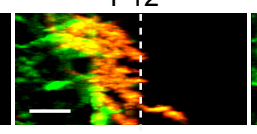

P14
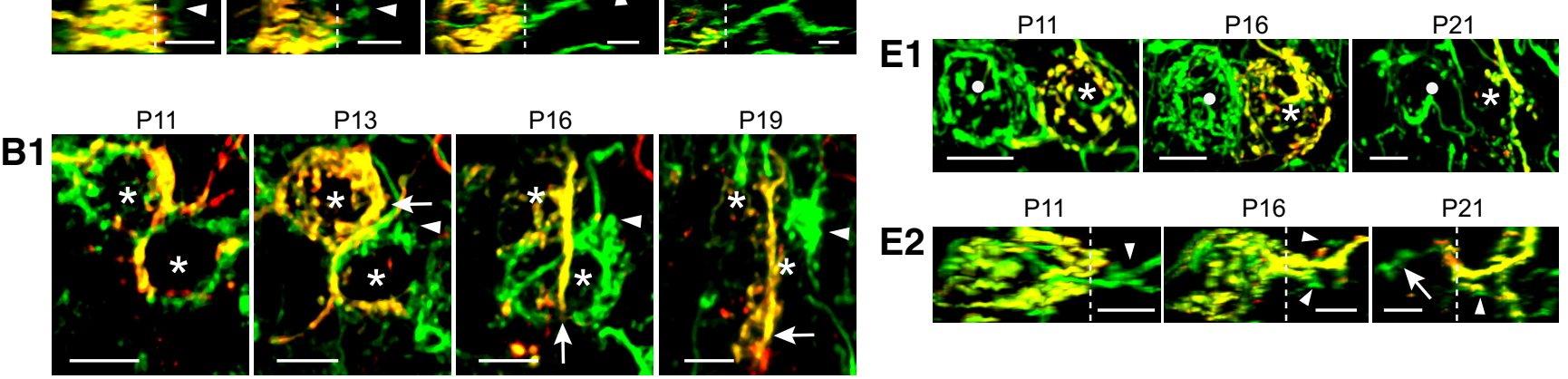

P16

$\mathrm{P} 19$
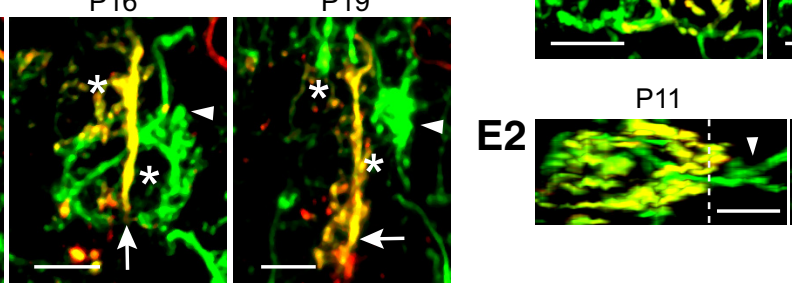

P16

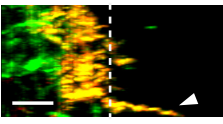

B2
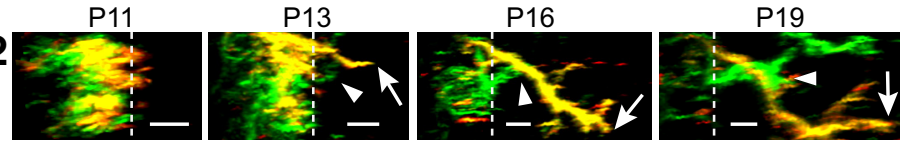

$\mathbf{F}$

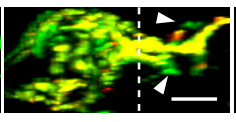

P21

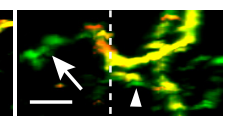

P11

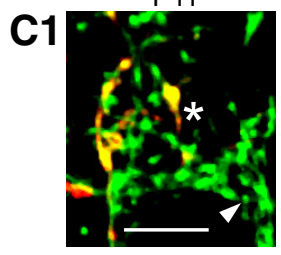

$\mathrm{P} 13$

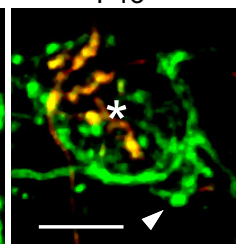

P15
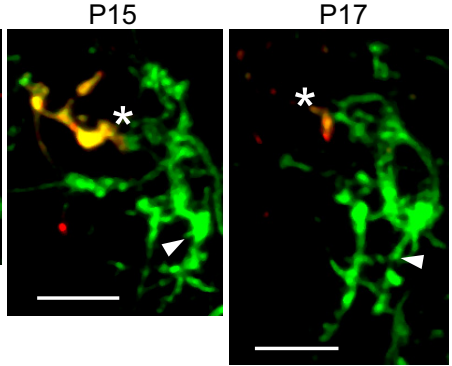

$\mathrm{P} 17$
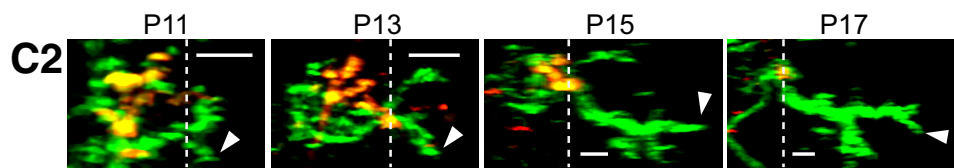

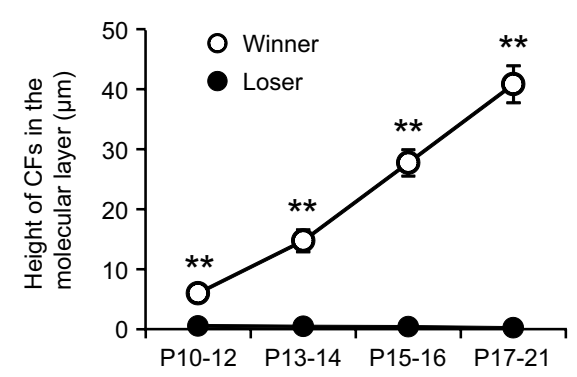

Figure 4. Time-lapse multicolor in vivo imaging of the same set of competing (Fs. CFs contain either EGFP only (green) or EGFP/TMR (yellow or orange). $A-D$, Top (A1-D1), Maximum projections showing top-down views of CFs that involve both somatic and dendritic regions. Asterisks indicate PCs innervated by both green and yellow CFs. White circles indicate neighboring PCs. Bottom (A2-D2), Sagittal views of the same multiply innervated PCs (indicated by asterisks in A1-D1) reconstructed from the image stacks collected for the top parts. Whole CF trees (i.e., terminal portion of olivocerebellar axons between the $\mathrm{PC}$ layer and pial surface) are shown. White dashed lines indicate approximate location of the border between soma and proximal dendrites. Pial surface is located at the right side of the images. Arrowheads indicate corresponding points on the dendrites in the top and bottom. Since two adjacent PCs were innervated by multiple CFs in $\boldsymbol{B}$, arrows (for the top left PC) are used in addition to arrowheads (for the bottom right PC). In the case of $\boldsymbol{A}$ and $\boldsymbol{C}$, the green (F was the winner and translocated. In $\boldsymbol{B}$, the yellow (F was the winner for the top left $P C$ whereas the green $C F$ was the winner for the bottom right $P C$. In $\boldsymbol{D}$, a green $(F$ was dominant at $P 10$, but an orange $C F$ dominated the green $C F$ later and became the winner (flip-flop). $\boldsymbol{E}$, A rare example of dual-CF dendritic translocation found in one PC. Maximum projections (E1) and sagittal views (E2) are shown as explained above for $A-D$, except that (1) only a part of the CF tree (soma and proximal portion of dendrites) are shown for P21, (2) arrowheads indicate the dendritic portion of the green $C F$, and (3) an arrow indicates somatic varicosities of the green CF at P21. Note that the green CF first translocated to the dendrites, but the yellow CF later translocated as well. Although the yellow CF eventually innervated most of the dendritic surfaces, the green CF still remained on dendrites (arrowhead) and soma (arrow) even at P21. Scale bars: $10 \mu \mathrm{m}$ in all images. $\boldsymbol{F}$, The height of (Fs in the molecular layer was quantified and compared between winner and loser at each time point. Error bars indicate SEM. ${ }^{* *} p<0.001$ (Mann-Whitney $U$ test). Note that these measurements may not be as accurate as conventional histological analyses because of the poor axial resolution of two-photon microscopy and brain pulsation during image acquisition.

tion. However, the CF that underwent dendritic translocation (i.e., winner) was not always morphologically dominant before the onset of dendritic translocation (Fig. $4 A, D$ ), suggesting that flip-flop (a dominant CF was later replaced by its competitor) occurred in some cases.

To examine whether the morphologically dominant CF is functionally dominant as well, we used cerebellar slices and combined CF-EPSCs recording with $\mathrm{Ca}^{2+}$ imaging and morpholog- ical imaging, in which a $\mathrm{Ca}^{2+}$ signal was used to confirm that a recorded EPSC is evoked by an imaged CF (Fig. $5 A-E$ ). We injected a mixture of TMR-dextran and OGB1 ( $\mathrm{Ca}^{2+}$ indicator)dextran (instead of TMR-dextran alone) into the inferior olive of the nefl-EGFP tg mice. The CFs labeled by this mixture became TMR/OGB1/EGFP triple positive. Complementing our in vivo observation, we often found PCs that were co-innervated by TMR/OGB1/EGFP triple-positive CF (yellow or orange) and 
A

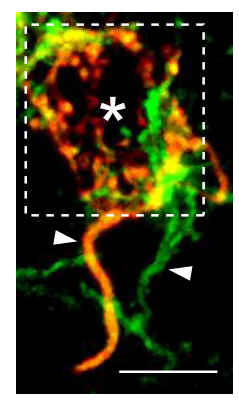

B
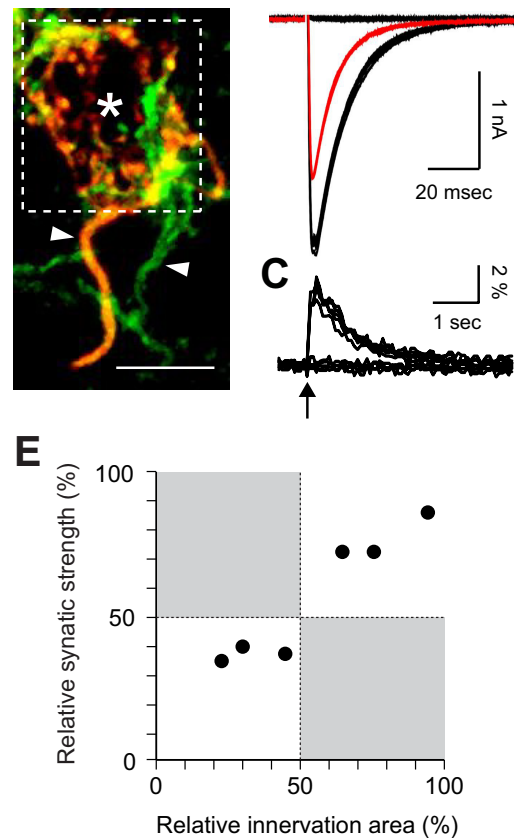

F

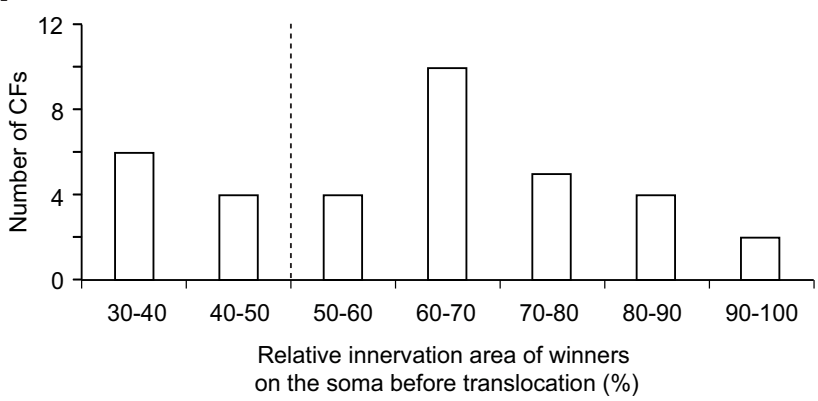

Figure 5. Functional analysis of morphologically identified CFs. A, A multicolor image of two competing (Fs in an acute cerebellar slice. The orange and green (Fs (arrowheads) innervate a PC (asterisk) before dendritic translocation. The green CF contains only EGFP and the orange $(F$ contains a calcium indicator (OGB1) in addition to the TMR and EGFP in the previous figures. Scale bar, $10 \mu \mathrm{m}$. B, CF-EPSCs were evoked by gradually increasing the stimulus intensity to $\mathrm{CF}$ axons in the granule cell layer. Traces are overlaid. $C$, Changes of green fluorescence upon CF stimulation were measured in the dashed box in $A$, simultaneously with the CF-EPSCs recordings. Traces were averaged three times. Arrow indicates the timing when the stimulation was given. D. Amplitude of EPSCS (top) and $\mathrm{Ca}^{2+}$ transient (bottom) as a function of stimulus intensity. The first EPSC step (red traces in $\boldsymbol{B}$ ) shared the same threshold as the $\mathrm{Ca}^{2+}$ signal, indicating that it was evoked by the orange CF (containing $\mathrm{Ca}^{2+}$ indicator). The second EPSC step was not accompanied by an increase in the $\mathrm{Ca}^{2+}$ signal because this step was the green $\mathrm{CF}$ (not containing $\mathrm{Ca}^{2+}$ indicator). $\boldsymbol{E}$, Relative synaptic strength of TMR/OGB1/EGFP triple-positive CFs (a ratio of the first EPSC amplitude to the total EPSC amplitude) is plotted against their relative innervation area (a ratio of somatic area innervated by the TMR/OGB1/EGFP triple-positive (F to the both (Fs). Morphological dominance of a $\mathrm{CF}$ correlates with its functional dominance in white quadrants, whereas they are not correlated in gray quadrants. Note: Data obtained from all six $P C s$ analyzed are in the white quadrants. $\boldsymbol{F}$, The distribution of the eventual winner CFs as a function of their relative innervation area on the somata. Multicolor in vivo time-lapse images taken before dendritic translocation proceeded were analyzed. of 35 winner CFs, the relative innervation area of $10 \mathrm{CFs}$ was $<50 \%$ (a dashed line in the histogram) on the somata before translocation, suggesting that flip-flop occurred.

EGFP single-positive CF (green) in acutely prepared cerebellar slices derived from P9-P10 mice (Fig. 5A). Whole-cell voltageclamp recordings were made from such $\mathrm{PCs}$ and simultaneous CF-EPSC recording and $\mathrm{Ca}^{2+}$ imaging were performed. A stimulation electrode was placed on the TMR/OGB1/EGFP triple- positive $\mathrm{CF}$ and stimulus intensity was gradually increased. As shown in Figure 5, B-D, both CF-EPSC and presynaptic $\mathrm{Ca}^{2+}$ transient showed all-or-none responses, indicating that they were caused by an action potential generated in the CF (Kreitzer et al., 2000). Since the thresholds for CF-EPSCs and $\mathrm{Ca}^{2+}$ signals were identical (Fig. 5D, top and bottom), we could confirm that the recorded EPSC is indeed evoked by the TMR/OGB1/EGFP triplepositive CF. When stimulus intensity was further increased, the second EPSC step was observed, indicating that another CF (EGFP single-positive CF) innervating the same $\mathrm{PC}$ was stimulated (Fig. $5 \mathrm{~B}, \mathrm{D}$; but there was no further increase of $\mathrm{Ca}^{2+}$ signals because this $\mathrm{CF}$ did not contain $\mathrm{Ca}^{2+}$ indicator). For example, in Figure 5, we could confirm that the PC was innervated by two CFs and the orange CF (TMR/OGB1/EGFP triple positive) produced $1.55 \mathrm{nA}$ of EPSC (the first EPSC step in Fig. $5 B$ ). Simultaneous activation of the orange CF and the green CF (EGFP single positive) produced $2.2 \mathrm{nA}$ of EPSC.

Of seven PCs recorded, one showed a third EPSC step, thus it was excluded from subsequent analysis. The other six PCs all showed two EPSC steps indicating that they were innervated by two CFs (i.e., each color represents a single CF). We therefore determined in these six PCs whether the relative innervation area of a TMR/OGB1/EGFP triple-positive CF [defined as a ratio of innervation area between a yellow CF and both CFs (green and yellow)] correlated with their relative synaptic strength (defined as a ratio of the first CF-EPSC amplitude to the total EPSC amplitude). As shown in Figure 5E, morphological dominance of TMR/OGB1/EGFP triple-positive CFs always correlated with their functional dominance.

We next quantified the relative innervation area of competing CFs in our multicolor in vivo images (all 35 PCs were analyzed). Relative innervation area of eventual winner CF on PC soma varied from $30 \%$ to almost $90 \%$ of total innervation area before dendritic translocation proceeded (Fig. $5 F$ ). Among them, flipflop (i.e., relative innervation area of eventual winner was $<50 \%$ ) was observed in 10 PCs ( $29 \%$ of the 35 PCs). It is estimated that only one or two of these 10 PCs might be innervated by three or more CFs at the time of analysis [P9 (three PCs), P10 (three PCs), and P11 (four PCs)] because we showed that CF triple innervation is rare at P9-P10 (by simultaneous CF-EPSCs recording and $\mathrm{Ca}^{2+}$ imaging) and P11 (by CF-EPSCs recording). Therefore, these results indicate that, although morphologically (and functionally) dominant CFs have a higher chance to win the competition, the dominance before dendritic translocation is not a decisive factor to determine the winner. However, once a CF begins translocation, this translocating $\mathrm{CF}$ becomes the winner.

\section{Selective photo-ablation of winning CFs reverses the fate of losing CFs}

Our results clearly showed that only a winning CF translocated to the dendrites of each PC, but it was unclear whether the winner was predetermined before translocation or translocation itself was the process of determining the winner versus loser. To clarify this, we performed in vivo selective photo-ablation of the winning $\mathrm{CF}$ (the CF that was undergoing dendritic translocation). We reasoned that if the winner was irreversibly determined before translocation, its ablation should not change the fate of the losing CFs. On the other hand, if translocation itself was the process of determining the winner versus loser, the fate of the losing CF would be expected to be reversed by ablating its winning competitor. 
Intense photo-excitation of fluorescent dyes has been used to selectively kill all or part of dye-filled neurons without affecting nearby nonlabeled cells (Miller and Selverston, 1979; Jacobs and Miller, 1985; Camhi and Macagno, 1991; Warzecha et al., 1992). It has been suggested that production of heat (through photoabsorption) and a toxic substance (through photodecomposition) by overexcitation causes this selective killing and any photo-absorptive dyes might allow selective killing of dye-filled cells upon intense excitation of the dyes (Miller and Selverston, 1979). We therefore thought that TMR-containing CFs (i.e., those containing EGFP/TMR) could be killed without affecting nearby EGFP single-positive CFs (i.e., those containing only EGFP), if TMR (but not GFP) could be selectively overexcited. However, at least within a range of two-photon excitation between 800 and $890 \mathrm{~nm}$, we could not find a condition in which TMR is selectively overexcited (data not shown). We then used A594 (instead of TMR) and found that A594-containing CFs can be selectively overexcited with little or minor excitation of EGFP at $800-830 \mathrm{~nm}$ (Fig. 6A, left). Importantly, the average laser power required for overexcitation of A594-containing CFs $(\sim 30$ $\mathrm{mW}$ at $830 \mathrm{~nm}$ ) was much less than that used for multicolor time-lapse imaging $(40-80 \mathrm{~mW}$ at $870-890 \mathrm{~nm})$. This is most likely because of the following: (1) A594 has higher two-photon cross sections than EGFP at $830 \mathrm{~nm}$ and (2) the number of dextran-conjugated fluorescent molecules (A594 in this case) is significantly more than that of EGFP in CFs (see Materials and Methods).

We next tested whether the $830 \mathrm{~nm}$ excitation could selectively ablate A594-containing CFs without affecting nearby EGFP single-positive CFs. Since the fluorescent signal of A594 alone is weak at $870-890 \mathrm{~nm}$ (Fig. $6 A$, right), it is not useful for simultaneous multicolor imaging with EGFP. We therefore injected a mixture of TMR-dextran and A594-dextran (instead of TMR or A594-dextran alone) into the inferior olive of nefl-EGFP tg mice and performed time-lapse in vivo imaging (Fig. 6B). The CFs labeled by this mixture became A594/EGFP/TMR triple positive. At the first imaging session, A594/EGFP/TMR triple-positive CFs and EGFP single-positive CFs were simultaneously imaged at $870-890 \mathrm{~nm}$, and then the entire field of view was illuminated at $830 \mathrm{~nm}$ for the overexcitation of A594. Multicolor imaging at $870-890 \mathrm{~nm}$ was then resumed from the next imaging session. As shown in Figure 6B, the A594/EGFP/TMR triple-positive CFs often showed an abnormal beaded morphology (typical for photo-damaged neuritis) at the next imaging session and the beaded CFs disappeared during the following imaging sessions. On the other hand, nearby EGFP-single positive CFs (including the one that was innervating the same PC as the A594/EGFP/ TMR triple-positive CF) were not affected even though they received exactly the same amount of laser illumination throughout the entire imaging sessions. This selective loss of A594/EGFP/ TMR triple-positive CFs was not due to bleaching of their fluorescence because of the following: (1) not only A594, but also EGFP signals disappeared in A594/EGFP/TMR triple-positive CFs, despite that EGFP was barely excited at $830 \mathrm{~nm}$; (2) A594/ EGFP/TMR triple-positive CFs often showed a sign of photo damage before they disappeared; and (3) fluorescence of A594/ EGFP/TMR triple-positive CFs did not recover at all. The entire intracellular space of the olivocerebellar axon (from the inferior olive located at the ventral surface of the medulla to the cerebellar cortex) is filled with fluorescent molecules, and we illuminated only its terminal (i.e., CF). Therefore, fluorescence should recover by intracellular diffusion of the fluorescent molecules, if bleaching accounted for the apparent loss of A594/EGFP/TMR triple-positive CFs. Indeed, it takes only $2 \mathrm{~d}$ for dextran conjugated fluorescent tracer to diffuse from the inferior olive to the terminal CF (Kreitzer et al., 2000). Moreover, fluorescence in axon terminals is known to recover quickly (in minutes) after photo-bleaching as shown at the NMJs (Turney and Lichtman, 2012).

It is unlikely that our photo-ablation killed the entire olivocerebellar axon. Rather, it locally damaged a segment of the axon (in our case, it was the CF) that was illuminated as shown in previous studies (Miller and Selverston, 1979; Jacobs and Miller, 1985). Regardless, our data indicate that innervation of PCs by A594-containing CFs can be selectively denervated during the period when CFs are competing. We therefore applied this photo-ablation technique when (1) two CFs were competing for the same $\mathrm{PC}$ and (2) one of them underwent dendritic translocation (Fig. $6 C, D$ ). Multicolor time-lapse imaging of two competing CFs was performed as described above at $870-890 \mathrm{~nm}$. As an A594/EGFP/TMR triple-positive CF (yellow or orange) began to translocate to the dendrites (becoming the winning $\mathrm{CF}$ ) and its competitor (EGFP single positive, green) remained at the soma (destined to be the losing CF), the winning A594/EGFP/TMR triple-positive CF was photo-ablated by overexcitation of A594 at $830 \mathrm{~nm}$. The same CFs were then imaged again at $870-890 \mathrm{~nm}$ every 2-3 d after the photo-ablation. We found that ablation of the winning CF induced growth of its competitor (in the case of 7 of 10 PCs). In these seven cases, the apparent losing CF (nonablated $\mathrm{CF}$ ) translocated to the dendrites and appeared to acquire the normal structure of mature CFs (Fig. 6C,D). Like the winning CF under normal circumstances (Fig. $4 F$ ), the height of the nonablated $\mathrm{CF}$ in the molecular layer linearly increased (Fig. 6E); the average height of the nonablated CF at later time points (P17P21) was $22 \mu \mathrm{m}$ higher than that immediately after photoablation (P13-P14). Although this increase was slightly smaller than that of the winning CF without photo-ablation $(29 \mu \mathrm{m})$, the difference between the two groups was not significant $(p>0.25$, Mann-Whitney $U$ test), indicating that photo-ablation turned the apparent losing CF into the winner (flip-flop). Without photo-ablation, the flip-flop was very rare after dendritic translocation. This significant difference in occurrence of flip-flop after dendritic translocation ( 7 of 10 PCs under photo-ablation vs 1 of 35 PCs under normal circumstance, $p<0.001$, Fisher's exact probability test) further indicates that A594-containing CFs were not just bleached. Flip-flop should not occur if the A594containing CFs just became invisible by bleaching (but they still existed). These results therefore indicate that the fate of losing CFs is not irreversibly determined before dendritic translocation. Rather, translocation itself is the process of determining the winner versus loser at least in some PCs.

\section{Discussion}

To our knowledge, this study is the first to use in vivo time-lapse microscopy to image synaptic competition and elimination in the developing CNS. We found that (1) during the period of late phase CF elimination, the terminals of CFs that are on the dendrites of individual PCs are more stable than those on the somata of the same PCs; (2) the fate of competing CFs was ultimately determined when one of them (the winner) began translocation to the dendrites; and (3) this dendritic translocation of the winner was not just a passive consequence of competition, but the active procedure to secure the "winner" status of the translocating CF. These results indicate that dendritic translocation is a key cellular event for late phase CF elimination. 
$830 \mathrm{~nm}, 30 \mathrm{~mW}$

A
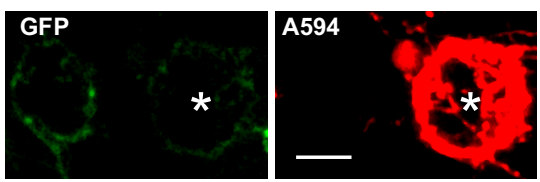

P8

B
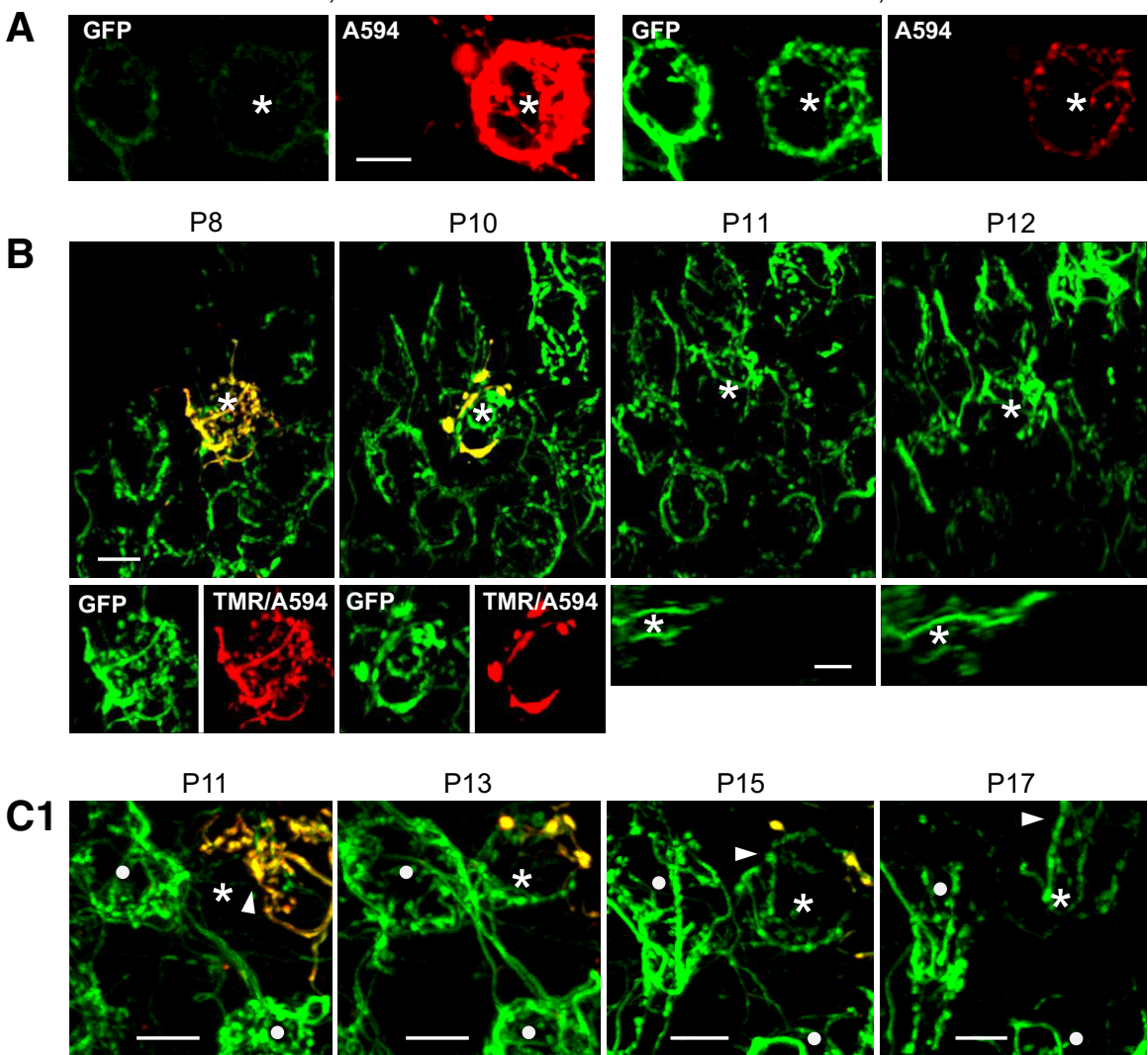

P11

C2
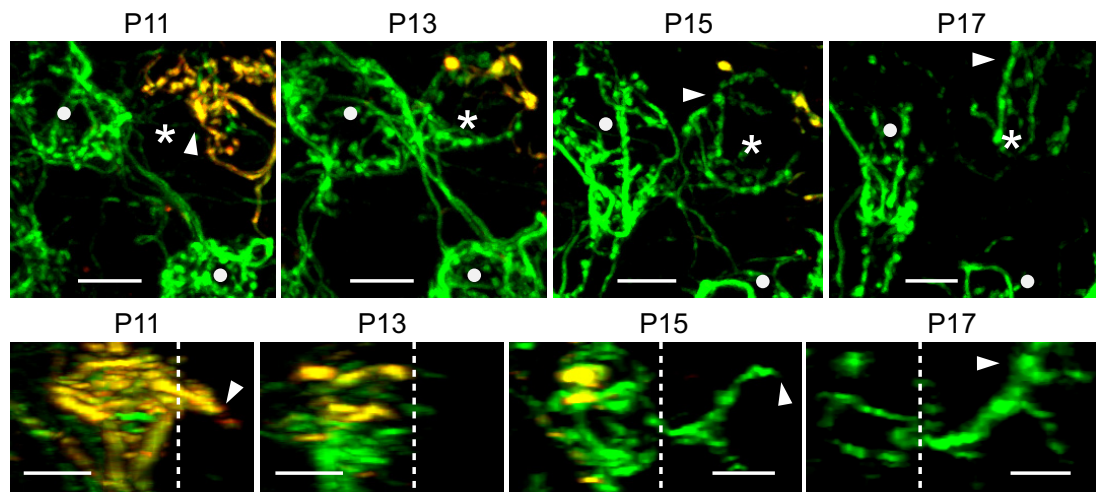

$\mathrm{P} 13$
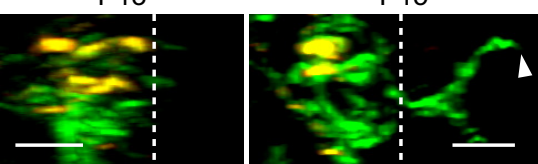

P11

P13

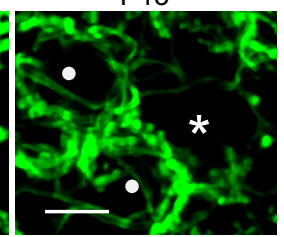

P13

D2

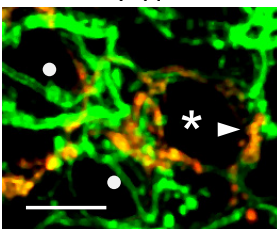

P11
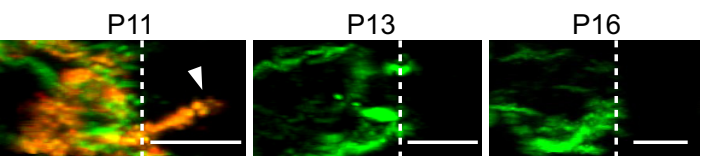

P16
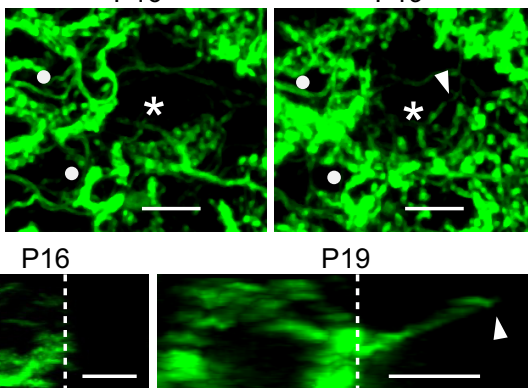

E

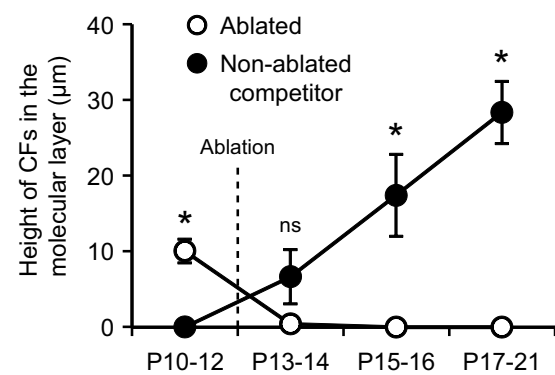

Figure 6. Selective photo-ablation of the "winning" CF (i.e., translocating onto the dendrite) causes the "losing CF" (i.e., nontranslocating) to translocate. $A, A 594$ can be selectively overexcited with little excitation of EGFP at $830 \mathrm{~nm}$. CFs contain either EGFP only or A594/EGFP. Asterisks indicate a PC innervated by the A594/EGFP double-positive CF. Green and red channels are individually shown. Left, CFs were excited at $830 \mathrm{~nm}$ with $30 \mathrm{~mW}$ of average laser power. Right, The same field of view was excited at $890 \mathrm{~nm}$ with $75 \mathrm{~mW}$ of average laser power. The detector setting was identical between green and red channels and the setting
Dendritic CF terminals in mature animals and somatic CF terminals in immature animals have quite different stability. More than $90 \%$ of the former are stable over a week (Nishiyama et al., 2007), whereas almost all of the latter disappear as dendritic translocation proceeds. However, before this study, it was unclear

was unchanged between 830 and $890 \mathrm{~nm}$ excitation. $\boldsymbol{B}, 0$ verexcitation of $A 594$ at $830 \mathrm{~nm}$ enabled selective photo-ablation of A594-containing CFs in vivo. CFs contain either EGFP only (green) or A594/EGFP/TMR (yellow). Only one (F in this image is A594/EGFP/TMR triple positive. All images were taken by multicolor excitation at $870-890 \mathrm{~nm}$. Top, Maximum projections showing top-down views of the CFs. At P8, a PC (asterisk) was innervated by both green and yellow CFs but the yellow CF was dominant. After taking the image, $830 \mathrm{~nm}$ laser light was delivered to the entire imaging field for selective overexcitation of A594. Two days later (P10), the yellow CFlost fine structural details and had several unusually big swellings: beaded structures that are typical for damaged neurites. The yellow CF disappeared at P11. Bottom, P8 and P10 show higher magnification views of red and green channels. P11 and P12 show sagittal views of the $P C$ (asterisk) innervated by the yellow $C F$ until P10. A green CF that co-innervated the PC was not ablated and showed developmental growth, including growth onto dendrites obvious in the P12 sagittal view. Note: EGFP signals near the bottom of the P12 image (top) are weak because of slight bone regeneration under the cranial window. $C$, $D$, Selective photo-ablation of the winning (translocating) CFs. CFs contain either EGFP only (green) or A594/EGFP/TMR (yellow or orange). All images were taken by multicolor excitation at $870-890 \mathrm{~nm}$. C1, D1, Maximum projections showing topdown views of the (Fs. At P11, PCs (asterisks) were innervated by both yellow/orange (winning) and green (losing) CFs. Neighboring PCs are indicated by white circles. C2, D2, Sagittal views of the same multiply innervated PCs (indicated by asterisks in $\mathbf{C 1}$ and D1) reconstructed from the image stacks collected for $\mathbf{C} \mathbf{1}$ and $\mathbf{D} \mathbf{1}$, respectively. Whole $\mathrm{CF}$ trees (i.e., terminal portion of olivocerebellar axons between the $\mathrm{PC}$ layer and pial surface) are shown. White dashed lines indicate approximate location of the border between the soma and proximal dendrite. Pial surface is located at the right side of the images. Arrowheads indicate corresponding points on the dendrites in the top-down views $(\mathbf{C 1}, \mathbf{D 1})$ and sagittal views $(\mathbf{C}, \mathbf{D 2})$. Scale bars: $10 \mu \mathrm{m}$ in all images. Note: The yellow/ orange CFs were already translocating to the dendrites at P11. Selective photo-ablation of these yellow/orange CFs was performed after taking the images at P11. In C, the yellow CF lost fine structural details at P13 and had several big swellings: beaded structures that are typical for damaged neurites. In contrast, the green $(F$ expanded its territory on the somata and later translocated to the dendrite (P15 and 17). In $\boldsymbol{D}$, the orange CF disappeared at P13. The green CF slowly expanded its territory on the somata and later translocated to the dendrite (P19). $\boldsymbol{E}$, The height of (Fs in the molecular layer was quantified and compared between the ablated CF (presumable winner under normal circumstances) and its nonablated competitor. The left and right side of the dashed line indicate before and after photo-ablation, respectively. Error bars indicate SEM. Asterisks and "ns" denote $p<0.01$ and $p>0.05$, respectively (Mann-Whitney $U$ test). Note that, immediately after photo-ablation (P13-P14), the difference between ablated and nonablated CFs was not significant, because 4 of 7 nonablated CFs had not yet begun dendritic translocation. 
whether somatic and dendritic CF terminals had different stabilities at the same developmental stage. We have shown that more than half of the somatic terminals appeared or disappeared within $1 \mathrm{~d}$, whereas a significantly smaller fraction $(\sim 20 \%)$ of the dendritic terminals, impinging on the same PCs, did so at approximately $\mathrm{P} 12$. This suggests that the characteristic lifetime of somatic and dendritic CF terminals during dendritic translocation is $\sim 1 \mathrm{~d}$ and $\geq 5 \mathrm{~d}$, respectively, and that dendritic translocation contributes to the stabilization of CF terminals. Since the proximal portion of the dendrites provides smaller postsynaptic surface area than the soma, a CF with stable terminals on dendrites may have a significant competitive advantage over its competitor.

Regarding the number of CFs that can translocate to the dendrites, previous studies (Scelfo et al., 2003; Hashimoto et al., 2009a) yielded conflicting results as described (see Results). Here, we repeatedly imaged the same set of competing CFs in vivo and found that only one CF could translocate to the dendrites in 34 of the 35 PCs analyzed. Dual-CF dendritic translocation, observed in only one PC, appeared to result in persistent multiple innervations. Our experiments not only confirmed the study by Hashimoto et al. (2009a), but also revealed for the first time how the winning and losing CFs sequentially develop in individual PCs. It has been shown that functional differentiation among competing CFs yields one strong and several weak inputs by P7, suggesting that the winner is predetermined before dendritic translocation (Hashimoto and Kano, 2003; but also see Scelfo and Strata, 2005). However, these results (obtained by comparing average characteristics of CF-EPSCs between different animals at different postnatal ages) represent overall progression of $\mathrm{CF}$ competition as a population, but not how the competition dynamically proceeds in individual PC. Therefore, before this study, it remained unknown whether or not the strongest CFs before translocation always became the winner. Only timelapse analysis of the same set of competing CFs can address this important question and our data show that the dominant CFs before translocation do not always win (i.e., flip-flop sometimes occurs). This new result, however, does not entirely contradict the idea that the winner is predetermined before translocation, because the dominant CFs before translocation have a higher chance to become the winners (Fig. $5 F$ ). Importantly, a CF that begins to translocate almost always wins the competition, suggesting that the dendritic translocation is the decisive factor to determine the winner during late phase CF elimination. This idea is further supported by the finding that photo-ablating a translocating (winning) CF reverses the fate of its losing competitor.

Kano and coworkers propose that dendritic translocation segregates innervation fields of winning and losing CFs between dendrites and soma and that the anatomical separation is essential for removal of the losing CFs (Hashimoto et al., 2009a; Watanabe and Kano, 2011). Our work does not test this particular model, but it supports the underlying idea that dendritic translocation is crucial for late phase elimination. However, experimental evidence suggests that not all the PCs require late phase elimination. Early phase CF elimination (which was not studied here) is driven by $\mathrm{P} / \mathrm{Q}$-type voltage-gated calcium channels on PCs and it removes some redundant CFs before dendritic translocation begins (Hashimoto et al., 2011). Late phase elimination is driven by activation of protein kinase $\mathrm{C} \gamma(\mathrm{PKC} \gamma)$ in PCs, and it removes the remaining redundant CFs as dendritic translocation proceeds (Kano and Hashimoto, 2009; Watanabe and Kano, 2011). A previous study showed that CF single inner- vation was established in $\sim 60 \%$ of PCs in PKC $\gamma$-deficient mice (Kano et al., 1995), indicating that late phase elimination is not necessary for all PCs. Nevertheless, late phase elimination is crucial for proper motor development as lack of this mechanism results in ataxia (Aiba et al., 1994; Chen et al., 1995; Offermanns et al., 1997). Perhaps, late phase elimination might function as a safety mechanism to ensure that almost all PCs can establish CF single innervation even when a few equally competitive CFs survive early phase elimination. CF single innervation is known to be established without dendritic translocation in cultured hindbrain explant and in "hyperspiney Purkinje cell" mutant mice (Sotelo, 1990; Letellier et al., 2009). It is therefore interesting to examine whether early phase elimination is augmented and/or prolonged in these cases to compensate for the lack of dendritic translocation.

The inability to image early phase elimination is one of the technical limitations of our imaging system: two-colors are not sufficient to distinguish all individual competing CFs during early phase. Combining our time-lapse imaging with the Brainbow technique (Livet et al., 2007) will allow in vivo time-lapse analysis of early phase elimination, and will provide significant insight into how the winner and loser are differentiated before dendritic translocation. Another technical concern is that our ablation experiment uses phototoxicity, a process that is not fully understood. Although phototoxicity-based selective cell ablation has been used successfully in various preparations (Miller and Selverston, 1979; Jacobs and Miller, 1985; Camhi and Macagno, 1991; Warzecha et al., 1992), damaging a focal spot in the tissues with high-power laser is a more commonly used technique to achieve selective axonal damage (Yanik et al., 2004). However, this method is not suitable in our experiments, because highpower laser might damage nearby competing CFs, PCs, and/or granule cells, all of which are important regulators for $\mathrm{CF}$ elimination (Cesa and Strata, 2009; Kano and Hashimoto, 2009; Hashimoto et al., 2009b; Watanabe and Kano, 2011). Recently, phototoxicity-based laser axotomy was successfully performed at the NMJs to ablate selective motor axons (Turney and Lichtman, 2012). They achieved the selectivity by confining the volume of two-photon excitation, while we did so by selectively overexciting A594. Thus, phototoxicity by two-photon excitation appears to be a promising tool for inducing selective neuronal damages.

Developmental synapse elimination shares a significant degree of similarity between the CF-PC synapses and the NMJ (Draft and Lichtman, 2009). The present study has revealed previously unrecognized similarities between the two systems as well as some potential differences. First, at the level of individual synapses, elimination does not proceed monotonically in either system. As reported at the NMJ (Walsh and Lichtman, 2003), flip-flop occurs at the CF-PC synapses and eventual losers often expand their innervation field until relatively late in the process of competition (Fig. 4). Second, deletion of winning inputs can reverse the fate of losing inputs in both systems. This suggests that the idea that synapse removal is driven by local interaction between competing inputs is widely applicable across the nervous system (Turney and Lichtman, 2012). Third, as competing motor axons segregate their territories at the NMJ (Walsh and Lichtman, 2003), the innervation fields of two competing CFs are often segregated at the soma before the onset of dendritic translocation (Fig. $4 A-C)$. Such segregation might be important to determine which CF translocates to the dendrites.

One difference between the two systems is that although retraction bulbs (degenerating tips of losing axons) are common at 
the NMJ (Bishop et al., 2004), we found such structures in only one PC (data not shown). This observation might mean that the retraction bulb of a losing CF is just difficult to detect, but this is similar to the previous study indicating that developmental pruning of axonal branches is not accompanied by the formation of retraction bulbs in the neocortex (Portera-Cailliau et al., 2005). Another potential difference is that late phase CF elimination appears to use distinct subcellular domains (soma and dendrites) of postsynaptic surfaces to determine winners versus losers. Such a mechanism might represent a neuron-neuron synapse or CNS synapse-specific safety device to ensure completion of synapse elimination.

Emerging evidence suggests that each neuronal subcellular domain serves as a distinct functional unit in regulating neuronal excitability and synaptic plasticity in the mature CNS (Mainen and Sejnowski, 1996; Govindarajan et al., 2006; Murakoshi and Yasuda, 2012). Our findings may expand the concept of this subcellular domain-specific regulation to CNS development.

\section{References}

Aiba A, Kano M, Chen C, Stanton ME, Fox GD, Herrup K, Zwingman TA, Tonegawa S (1994) Deficient cerebellar long-term depression and impaired motor learning in mGluR1 mutant mice. Cell 79:377-388. CrossRef Medline

Bishop DL, Misgeld T, Walsh MK, Gan WB, Lichtman JW (2004) Axon branch removal at developing synapses by axosome shedding. Neuron 44:651-661. CrossRef Medline

Camhi JM, Macagno E (1991) Using fluorescence photoablation to study the regeneration of singly cut leech axons. J Neurobiol 22:116-129. CrossRef Medline

Cesa R, Strata P (2009) Axonal competition in the synaptic wiring of the cerebellar cortex during development and in the mature cerebellum. Neuroscience 162:624-632. CrossRef Medline

Chen C, Kano M, Abeliovich A, Chen L, Bao S, Kim JJ, Hashimoto K, Thompson RF, Tonegawa S (1995) Impaired motor coordination correlates with persistent multiple climbing fiber innervation in PKC gamma mutant mice. Cell 83:1233-1242. CrossRef Medline

Crepel F, Mariani J, Delhaye-Bouchaud N (1976) Evidence for a multiple innervation of Purkinje cells by climbing fibers in the immature rat cerebellum. J Neurobiol 7:567-578. CrossRef Medline

Draft RW, Lichtman JW (2009) It's lonely at the top: winning climbing fibers ascend dendrites solo. Neuron 63:6-8. CrossRef Medline

Forehand CJ (1985) Density of somatic innervation on mammalian autonomic ganglion cells is inversely related to dendritic complexity and preganglionic convergence. J Neurosci 5:3403-3408. Medline

Fremeau RT Jr, Troyer MD, Pahner I, Nygaard GO, Tran CH, Reimer RJ, Bellocchio EE, Fortin D, Storm-Mathisen J, Edwards RH (2001) The expression of vesicular glutamate transporters defines two classes of excitatory synapse. Neuron 31:247-260. CrossRef Medline

Govindarajan A, Kelleher RJ, Tonegawa S (2006) A clustered plasticity model of long-term memory engrams. Nat Rev Neurosci 7:575-583. CrossRef Medline

Hashimoto K, Kano M (2003) Functional differentiation of multiple climbing fiber inputs during synapse elimination in the developing cerebellum. Neuron 38:785-796. CrossRef Medline

Hashimoto K, Ichikawa R, Kitamura K, Watanabe M, Kano M (2009a) Translocation of a "winner" climbing fiber to the Purkinje cell dendrite and subsequent elimination of "losers" from the soma in developing cerebellum. Neuron 63:106-118. CrossRef Medline

Hashimoto K, Yoshida T, Sakimura K, Mishina M, Watanabe M, Kano M (2009b) Influence of parallel fiber-Purkinje cell synapse formation on postnatal development of climbing fiber-Purkinje cell synapses in the cerebellum. Neuroscience 162:601-611. CrossRef Medline

Hashimoto K, Tsujita M, Miyazaki T, Kitamura K, Yamazaki M, Shin HS, Watanabe M, Sakimura K, Kano M (2011) Postsynaptic P/Q-type Ca2+ channel in Purkinje cell mediates synaptic competition and elimination in developing cerebellum. Proc Natl Acad Sci U S A 108:9987-9992. CrossRef Medline

Holtmaat A, Bonhoeffer T, Chow DK, Chuckowree J, De Paola V, Hofer SB, Hübener M, Keck T, Knott G, Lee WC, Mostany R, Mrsic-Flogel TD,
Nedivi E, Portera-Cailliau C, Svoboda K, Trachtenberg JT, Wilbrecht L (2009) Long-term, high-resolution imaging in the mouse neocortex through a chronic cranial window. Nat Protoc 4:1128-1144. CrossRef Medline

Hume RI, Purves D (1981) Geometry of neonatal neurones and the regulation of synapse elimination. Nature 293:469-471. CrossRef Medline

Ichikawa R, Miyazaki T, Kano M, Hashikawa T, Tatsumi H, Sakimura K, Mishina M, Inoue Y, Watanabe M (2002) Distal extension of climbing fiber territory and multiple innervation caused by aberrant wiring to adjacent spiny branchlets in cerebellar Purkinje cells lacking glutamate receptor delta 2. J Neurosci 22:8487-8503. Medline

Jacobs GA, Miller JP (1985) Functional properties of individual neuronal branches isolated in situ by laser photoinactivation. Science 228:344-346. CrossRef Medline

Kano M, Hashimoto K (2009) Synapse elimination in the central nervous system. Curr Opin Neurobiol 19:154-161. CrossRef Medline

Kano M, Hashimoto K, Chen C, Abeliovich A, Aiba A, Kurihara H, Watanabe M, Inoue Y, Tonegawa S (1995) Impaired synapse elimination during cerebellar development in PKC gamma mutant mice. Cell 83:1223-1231. CrossRef Medline

Kano M, Hashimoto K, Kurihara H, Watanabe M, Inoue Y, Aiba A, Tonegawa S (1997) Persistent multiple climbing fiber innervation of cerebellar Purkinje cells in mice lacking mGluR1. Neuron 18:71-79. CrossRef Medline

Katz LC, Shatz CJ (1996) Synaptic activity and the construction of cortical circuits. Science 274:1133-1138. CrossRef Medline

Kreitzer AC, Gee KR, Archer EA, Regehr WG (2000) Monitoring presynaptic calcium dynamics in projection fibers by in vivo loading of a novel calcium indicator. Neuron 27:25-32. CrossRef Medline

Letellier M, Wehrl é R, Mariani J, Lohof AM (2009) Synapse elimination in olivo-cerebellar explants occurs during a critical period and leaves an indelible trace in Purkinje cells. Proc Natl Acad Sci U S A 106: 14102-14107. Medline

Li M, Cui Z, Niu Y, Liu B, Fan W, Yu D, Deng J (2010) Synaptogenesis in the developing mouse visual cortex. Brain Res Bull 81:107-113. CrossRef Medline

Lichtman JW, Colman H (2000) Synapse elimination and indelible memory. Neuron 25:269-278. CrossRef Medline

Livet J, Weissman TA, Kang H, Draft RW, Lu J, Bennis RA, Sanes JR, Lichtman JW (2007) Transgenic strategies for combinatorial expression of fluorescent proteins in the nervous system. Nature 450:56-62. CrossRef Medline

Lohof AM, Delhaye-Bouchaud N, Mariani J (1996) Synapse elimination in the central nervous system: functional significance and cellular mechanisms. Rev Neurosci 7:85-101. Medline

Mainen ZF, Sejnowski TJ (1996) Influence of dendritic structure on firing pattern in model neocortical neurons. Nature 382:363-366. CrossRef Medline

Miller JP, Selverston A (1979) Rapid killing of single neurons by irradiation of intracellularly injected dye. Science 206:702-704. CrossRef Medline

Murakoshi H, Yasuda R (2012) Postsynaptic signaling during plasticity of dendritic spines. Trends Neurosci 35:135-143. CrossRef Medline

Nishiyama H, Fukaya M, Watanabe M, Linden DJ (2007) Axonal motility and its modulation by activity are branch-type specific in the intact adult cerebellum. Neuron 56:472-487. CrossRef Medline

Offermanns S, Hashimoto K, Watanabe M, Sun W, Kurihara H, Thompson RF, Inoue Y, Kano M, Simon MI (1997) Impaired motor coordination and persistent multiple climbing fiber innervation of cerebellar Purkinje cells in mice lacking Galphaq. Proc Natl Acad Sci U S A 94:14089-14094. CrossRef Medline

Palay S, Chan-Palay V (1974) Cerebellar cortex: cytology and organization. New York: Springer.

Personius KE, Balice-Gordon RJ (2001) Loss of correlated motor neuron activity during synaptic competition at developing neuromuscular synapses. Neuron 31:395-408. CrossRef Medline

Personius KE, Chang Q, Mentis GZ, O’Donovan MJ, Balice-Gordon RJ (2007) Reduced gap junctional coupling leads to uncorrelated motor neuron firing and precocious neuromuscular synapse elimination. Proc Natl Acad Sci U S A 104:11808-11813. CrossRef Medline

Portera-Cailliau C, Weimer RM, De Paola V, Caroni P, Svoboda K (2005) Diverse modes of axon elaboration in the developing neocortex. PLoS Biol 3:e272. CrossRef Medline 
Purves D, Lichtman JW (1980) Elimination of synapses in the developing nervous system. Science 210:153-157. CrossRef Medline

Ramon y Cajal S (1911) Histologie du Systeme Nerveux de l'Homme et des Vertebres. Paris: Maloine.

Scelfo B, Strata P (2005) Correlation between multiple climbing fibre regression and parallel fibre response development in the postnatal mouse cerebellum. Eur J Neurosci 21:971-978. CrossRef Medline

Scelfo B, Strata P, Knöpfel T (2003) Sodium imaging of climbing fiber innervation fields in developing mouse purkinje cells. J Neurophysiol 89: 2555-2563. CrossRef Medline

Sotelo C (1990) Cerebellar synaptogenesis: what we can learn from mutant mice. J Exp Biol 153:225-249. Medline

Sugihara I (2005) Microzonal projection and climbing fiber remodeling in single olivocerebellar axons of newborn rats at postnatal days 4-7. J Comp Neurol 487:93-106. CrossRef Medline

Sugihara I, Wu HS, Shinoda Y (2001) The entire trajectories of single olivocerebellar axons in the cerebellar cortex and their contribution to Cerebellar compartmentalization. J Neurosci 21:7715-7723. Medline
Turney SG, Lichtman JW (2012) Reversing the outcome of synapse elimination at developing neuromuscular junctions in vivo: evidence for synaptic competition and its mechanism. PLoS Biol 10:e1001352. CrossRef Medline

Walsh MK, Lichtman JW (2003) In vivo time-lapse imaging of synaptic takeover associated with naturally occurring synapse elimination. Neuron 37:67-73. CrossRef Medline

Warzecha AK, Borst A, Egelhaaf M (1992) Photo-ablation of single neurons in the fly visual system reveals neural circuit for the detection of small moving objects. Neurosci Lett 141:119-122. CrossRef Medline

Watanabe M, Kano M (2011) Climbing fiber synapse elimination in cerebellar Purkinje cells. Eur J Neurosci 34:1697-1710. CrossRef Medline

Yanik MF, Cinar H, Cinar HN, Chisholm AD, Jin Y, Ben-Yakar A (2004) Neurosurgery: functional regeneration after laser axotomy. Nature 432: 822. CrossRef Medline

Zipfel WR, Williams RM, Webb WW (2003) Nonlinear magic: multiphoton microscopy in the biosciences. Nat Biotechnol 21:1369-1377. CrossRef Medline 